\title{
Data-driven constitutive model for the inelastic response of metals:
}

\section{application to $316 \mathrm{H}$ steel}

Aaron E. Tallman ${ }^{1, *}$, M. Arul Kumar ${ }^{1}$, Andrew Castilloº, Wei Wen ${ }^{1}$, Laurent Capolungo ${ }^{1}$, Carlos

$$
\text { N. Tomé }{ }^{1}
$$

1. Materials Science and Technology Division, Los Alamos National Laboratory, Los Alamos,

$$
\text { NM, USA }
$$

2. George W. Woodruff School of Mechanical Engineering, Georgia Institute of Technology,

$$
\text { Atlanta, GA, USA }
$$

*Corresponding Author, aaront@lanl.gov

On behalf of all authors, the corresponding author states that there is no conflict of interest. 


\begin{abstract}
Predictions of the mechanical response of structural elements are conditioned by the accuracy of constitutive models used at the engineering length-scale. In this regard, a prospect of mechanistic crystal-plasticity-based constitutive models is that they could be used for extrapolation beyond regimes in which they are calibrated. However, their use for assessing the performance of a component is computationally onerous. To address this limitation, a new approach is proposed whereby a surrogate constitutive model (SM) of the inelastic response of $316 \mathrm{H}$ steel is derived from a mechanistic crystal plasticity-based polycrystal model tracking the evolution of dislocation densities on all slip systems. The latter is used to generate a database of the expected plastic response and dislocation content evolution associated with several instances of creep loading. From the database, a SM is developed. It relies on the use of orthogonal polynomial regression to describe the evolution of the dislocation content. The SM is then validated against predictions of the dead load creep response given by the polycrystal model across a range of temperatures and stresses. When the SM is used to predict the response of $316 \mathrm{H}$ during complex non monotonic loading, extrapolating to new loading conditions, it is found that predictions compare particularly well against those from the physics based polycrystal model.
\end{abstract}

Keywords: Crystal Plasticity, Reduced Order Modeling

\title{
1. Introduction
}

Structural design and certification of metallic components subjected to extreme environments (e.g. high stress, temperature, irradiation) necessarily relies on predictions of the evolution of stresses, elastic strains and inelastic strains during service. This is usually achieved via the use of finite element (FE)-based mechanical and thermal solvers in which the elastic and 
inelastic response of the polycrystalline metal is described by a constitutive model [1-6]. Ideally, this constitutive model should predict not only the monotonic response of the medium, but also its response under complex loading conditions (e.g. cycling loading, creep-fatigue) over a wide range of imposed stresses, strain rates and temperatures. In practice, multiple deformation mechanisms can simultaneously contribute to the inelastic response of metals [7-11]. For example, the relative contributions of dislocation glide, climb and Coble creep mechanisms to the creep response of HT9 alloy was studied for a wide range of imposed stress and temperature conditions [12]. This study shows that the dislocation-mediated deformation diminishes from the primary to the secondary creep regimes to the benefit of diffusion mediated plasticity. Further, the onset of transition from one mechanism to another is found to have a complex dependence on temperature, stress, and microstructure state [12]. Ideally, a constitutive model should capture these transitions. A key difficulty is that experimental data are usually scarce, particularly for high temperature creep applications [13-16]. Further, the mathematical form of the constitutive model must be simple in order to reduce computational costs. As a result, achieving both predictiveness and computational efficiency is challenging.

In general, two approaches have been employed to derive constitutive models: (i) phenomenological/empirical and (ii) crystal plasticity-based (CP) mechanistic models. The former approach does not describe the plastic deformation occurring in each individual crystal but rather proposes to directly describe the response of the polycrystal [17-25]. These methods are numerically efficient and have successfully been used to predict the deformation behavior of metals under monotonic loading, creep conditions [17,20,26-28], and cyclic loading [18,19,21,2932]. As previously mentioned, the relative contribution of deformation mechanisms changes with stress and temperature, and so the phenomenological/empirical model calibration will also change. 
For example, in the case of creep described with a simple power law, the coefficients and power law creep exponents may have to be changed from one stress-temperature regime to the other [33]. Absent any experimental data, it is unclear how accurate such approach would be in regimes far removed from the regime in which a model is calibrated.

In mechanistic constitutive model, the relative activation of distinct deformation mechanisms and the state of microstructure are tracked [12,34-44]. In principle, these methods should allow predicting the creep responses of metals for a range of stresses and temperatures going beyond the calibrated domain. Clearly the predictiveness in these regimes will necessarily be conditioned by that of the models describing each deformation mechanism [45,46]. However, the direct use of these $\mathrm{CP}$ based mechanistic constitutive models within the FE solver at every integration point of a structural component [47] is computationally cost-prohibitive [48-52].

Among others, surrogate models (SM) have been proposed as an alternative to computationally expensive high-fidelity models for a wide range of applications including process design and material modeling [53,54]. SMs are typically derived from databases of expected responses. Here, the expected response is the visco-plastic behavior of metals as a function of imposed stress and temperature, and microstructure. A SM can take the form of a simple look-up table, a simpler constitutive model calibrated against a high-fidelity model [55] or, as proposed in works of Kalidindi et al. [56,57], of a spectral Fourier series representation. These SMs have thus far been applied successfully to the prediction of plasticity and plastically induced texture changes in polycrystalline metals. As shown by Zecevic et al. [58], the use of spectral methods can lead to an acceleration of up to 100 times vis a vis numerically integrated schemes. While in strain-driven plastic deformation the load conditions drive the microstructure evolution, in the case of creep the initial and the evolving microstructure play a key role on the resulting strain response. As a 
consequence, creep predictions are very sensitive to details of the model used, and pose a more stringent benchmark on the model. To our knowledge, these SMs have not been developed to capture the response of structural materials under constant imposed stress and at high temperature.

In this work, we aim to develop a computationally efficient SM to predict the visco-plastic creep response of $316 \mathrm{H}$ for wide range of stresses and temperature. The primary objective of the work is to assess whether such a SM can predict the response of a polycrystal subjected to nonmonotonic loading. In this work, we employ recently developed CP-based mechanistic constitutive model [12] to generate a synthetic database of expected mechanical response which complements the experimentally reported data $[13,14]$. The synthetic database is critical to generating enough data from which a SM can be derived. The database is generated using a recently proposed CPbased constitutive model based on the Visco-Plastic Self Consistent (VPSC) framework, which is calibrated and validated using experimental data of $316 \mathrm{H}$ alloy. A database of plastic response and dislocation content evolution resolved over time during 11,674 simulations of creep loading across temperatures and stresses ranging from 800 to $1000 \mathrm{~K}$ and 120 to $300 \mathrm{MPa}$ is developed. Data reduction is performed to simplify the database to homogenized polycrystal response. The SM is developed from this database using orthogonal polynomial regression [59] to derive the datadriven constitutive equations and evolution equations for homogenized dislocation densities, and through these equations accurately capture deformation during plastic loading.

First, as a validation, the SM is applied to predict creep responses for a new set of stresses and temperatures and is compared with the mechanistic CP model predictions. Then, the model is applied to simulate three complex loading scenarios: (i) sudden stress jump during relaxation, (ii) stress cycling, (iii) thermal cycling. In all cases it is found that, despite the fact that the surrogate model was trained against monotonic dead load creep simulations, it can acceptably predict the 
response of $316 \mathrm{H}$ in non-monotonic loading. Discussions of the connection between the surrogate model and phenomenological models helps rationalize these findings.

\section{Methods}

The following steps are considered to derive the SM. First, a CP-based constitutive model is selected, calibrated and validated against experimental data to predict the creep behavior of $316 \mathrm{H}$ alloy. For the sake of completeness, the CP-model is briefly presented in Sec. 2.1. The calibration and validation of the model against experimental creep data of $316 \mathrm{H}$ alloy is presented in Sec. 2.2. Then, a synthetic database of homogenized creep responses of the polycrystal and state of microstructure is generated as a function of imposed stress and temperature in Sec. 2.3. Last, a predictive SM framework based on Legendre polynomial expansion is derived from the synthetic database in Sec. 2.4.

\subsection{Mechanistic constitutive model}

Creep and microstructure evolution data for SM development is generated using a crystal plasticity based constitutive model in a Visco-Plastic Self Consistent (VPSC) framework. The physics based constitutive model used was originally proposed by Wang et al. [60] and applied to face centered cubic [46], body centered cubic $[12,61]$ and hexagonal close packed metals $[62,63]$. In this work, the polycrystalline aggregate is homogenized using the affine formulation $[64,65]$. For the sake of completeness this constitutive model will be summarized in what follows.

The total creep rate is expressed as:

$$
\dot{\varepsilon}^{p}=\dot{\varepsilon}^{d}+\dot{\varepsilon}^{c}+\dot{\varepsilon}^{c o b l e}
$$


$\dot{\varepsilon}^{d}, \dot{\varepsilon}^{c}$ and $\dot{\varepsilon}^{\text {coble }}$ refer to the plastic deformation accumulated through dislocation glide, climb and Coble creep, respectively. The strain rate due to dislocation motion is written as the sum of the mean shear/climb rates contributed by all active slip systems in a given grain.

$$
\dot{\varepsilon}^{d}=\sum_{s} m_{i j}^{s} \overline{\dot{\gamma}}^{s} \text { and } \dot{\varepsilon}^{c}=\sum_{s} c_{i j}^{s} \dot{\bar{\beta}}^{s}
$$

Here $\boldsymbol{m}^{s}$ is the symmetric part of the Schmid tensor. $\boldsymbol{c}^{s}=\left(\boldsymbol{b}^{s} \otimes \boldsymbol{b}^{s}\right)$ is the climb tensor for edge dislocations [46] and $\overline{\dot{\gamma}}^{s}$ and $\overline{\dot{\beta}}^{s}$ denote the mean shear and climb rates in slip system $s$ of the grain, respectively. Coble creep contributes to plastic deformation in polycrystals via migration of point defects along the grain boundaries. According to Coble [45], the creep law depends on the grain boundary width and grain boundary diffusion coefficient of vacancies or interstitials. In the present model, grain boundaries are not represented explicitly, and grain boundary related material properties are not known for the steel alloy system considered. Thus, the Coble creep model is written in simple form as,

$$
\dot{\varepsilon}_{i j}^{\text {coble }}=\frac{A^{\text {coble }} \sigma_{i j}}{T} \exp \left(-\frac{Q_{g b}}{k T}\right)
$$

where $A^{\text {coble }}$ and $Q_{\mathrm{gb}}$ are effective parameters accounting for all point defects, which are obtained here through the fitting to the experimental data. $\sigma_{i j}$ denotes the $i j$ component of the deviatoric stress tensor. The hydrostatic component of stress is not included in the VPSC framework.

As per Wang et al. $[60,62]$, the model tracks the internal stress distribution in each grain, specifically intragranular residual stresses arising from the presence of dislocations (i.e., type III stresses). This is accomplished by defining a grain as a material point, in turn consisting of a set of sub-material points that reflect the distribution of stress values. The width of the distribution is 
directly related to dislocation content. Accordingly, the mean shear rate of the slip system $s$ in the VPSC grain is expressed as:

$$
\overline{\dot{\gamma}}^{s}=\int_{-\infty}^{\infty} \dot{\gamma}^{s}\left(\tau^{s}\right) P\left(\tau^{s}-\bar{\tau}^{s}\right) d \tau^{s}
$$

where $\dot{\gamma}^{S}$ is the shear rate of one sub-material point. $\tau^{s}$ is the local resolved shear stress. $\bar{\tau}^{s}=$ $\boldsymbol{\sigma}: \boldsymbol{m}^{s}$ denotes the mean resolved shear stress in one grain (assumed to be the same for all systems), where $\boldsymbol{\sigma}$ is the deviatoric stress tensor. Similarly, the mean climb rate on slip system s can be written as,

$$
\overline{\dot{\beta}}^{s}=\int_{-\infty}^{\infty} \dot{\beta}^{s}\left(\tau_{\text {climb }}^{S}\right) P\left(\tau_{\text {climb }}^{s}-\bar{\tau}_{\text {climb }}^{S}\right) d \tau_{\text {climb }}^{S}
$$

where $\tau_{\text {climb }}^{S}$ and $\bar{\tau}_{\text {climb }}^{S}=\boldsymbol{\sigma}: \boldsymbol{c}^{S}$ are the local and global resolved climb stress, respectively. At individual sub-material points, the glide and climb rates are explicitly connected to the dislocation density. The local shear rate on slip system $s$ at a sub-material point is expressed using Orowan's equation:

$$
\dot{\gamma}^{s}=\rho_{\text {cell }}^{s} b^{s} v^{s} \operatorname{sign}\left(\tau^{s}\right)
$$

here $\operatorname{sign}\left(\tau^{s}\right)$ defines the direction of the shear rate to be the same as the direction of glide. $b^{s}$ is the magnitude of the Burgers vector. $v^{s}$ is the mean dislocation velocity, which is calculated from the dislocation mean free path, $\lambda^{s}$, and the total time a dislocation spends traveling between obstacles, the details of which are contained in the Appendix. Similar to glide, climb rate is also expressed as an Orowan type equation:

$$
\dot{\beta}^{s}=\rho_{\text {cell, }, \text { edge }}^{s} b^{s} v_{\text {climb }}^{s}
$$


here $\rho_{\text {cell,edge }}^{S}$ denotes the edge dislocation density. In the present work $\rho_{\text {cell,edge }}^{S}=0.1 \rho_{\text {cell }}^{S}$ is assumed. $v_{\text {climb }}^{S}$ in Eq. 7 represents the climb velocity, which depends on the net flux of point defects $I^{s}$. The climb velocity $v_{\text {climb }}^{S}$ depends on the imbalance between vacancies and interstitials being trapped by the dislocation, as shown in detail in the Appendix.

The probability distribution function $P\left(\tau^{S}-\bar{\tau}^{s}\right)$, representing the volume fraction of submaterial points with local stress $\tau^{s}$, is a Gaussian and written as:

$$
P\left(\tau^{S}-\bar{\tau}^{S}\right)=\frac{1}{\sqrt{2 \pi V}} \exp \left(-\frac{\left(\tau^{S}-\bar{\tau}^{S}\right)^{2}}{2 V^{2}}\right)
$$

where $V$ is the distribution variance. Following Wang et al. [62], the dispersion of intragranular stress is expressed in terms of total dislocation density as:

$$
V=\eta \sqrt{\rho_{\text {cell }}+\rho_{c w}}
$$

where $\eta \sim 10^{7} \mathrm{MPa} / \mathrm{m}$ is an effective scaling coefficient, and $\rho_{c e l l}$ and $\rho_{c w}$ are the total dislocation densities in the cell (subgrain) interior and in the cell walls (subgrain boundaries), respectively. The equations defining the evolution of dislocation densities are presented in the Appendix, along with additional details of the glide and climb rate equations.

\subsection{Calibration and Validation to Experimental Data}

Thermal creep simulations of $316 \mathrm{H}$ steel are performed using the affine-VPSC formulation [65] for three different temperatures, namely, $650^{\circ} \mathrm{C}, 700^{\circ} \mathrm{C}$ and $750^{\circ} \mathrm{C}$. Four different stresses are considered for each temperature, as follows. For $650^{\circ} \mathrm{C}: 110 \mathrm{MPa}, 150 \mathrm{MPa}, 200 \mathrm{MPa}$ and $250 \mathrm{MPa}$; for $700^{\circ} \mathrm{C}: 75 \mathrm{MPa}, 100 \mathrm{MPa}, 150 \mathrm{MPa}$ and $200 \mathrm{MPa}$; and for $750^{\circ} \mathrm{C}: 75 \mathrm{MPa}, 100 \mathrm{MPa}, 150 \mathrm{MPa}$, 200MPa. The polycrystal model parameters are calibrated against the experimental results reported 
in $[13,14]$. The following five experimental cases were used for calibration: $650^{\circ} \mathrm{C}$ and $110 \mathrm{MPa}$, $650^{\circ} \mathrm{C}$ and $200 \mathrm{MPa}, 700^{\circ} \mathrm{C}$ and $100 \mathrm{MPa}, 750^{\circ} \mathrm{C}$ and $75 \mathrm{MPa}, 750^{\circ} \mathrm{C}$ and $100 \mathrm{MPa}$. The remaining cases, a total of 7, are predicted and validated against the experiment. The model material is assumed to be heat-treated; thus, the texture is approximated by 50 randomly oriented grains, as shown in Figure 1(a). The initial dislocation densities are not known. We choose the initial values of $\rho_{c e l l}^{S}$ and $\rho_{c w}^{S}$ for each system to be $1 \times 10^{13} \mathrm{~m}^{-2}$ and $5 \times 10^{11} \mathrm{~m}^{-2}$, respectively. The predicted and calibrated model parameters are listed in Table A1, included in the Appendix.

The creep behavior of $316 \mathrm{H}$ steel predicted by the model for the three temperature cases is shown in Fig. 1(b)-(d), along with the experimental data. It clearly shows that the polycrystal model captures the thermal creep behavior correctly for all stress and temperature cases. The success of the model predictions is contingent on correctly capturing the relative contribution of glide, climb and Coble creep modes to the overall creep rate. At the early state of creep and for all stress and temperature cases, mostly glide alone contributes to the creep rate. After a few hours as dislocations are depleted due to annihilation, the contribution of glide decreases rapidly, while the Coble creep modes start to dominate. The contribution of the climb mode is relatively low compared to glide and Coble creep, although its relative activity increases with increasing temperature. Refer to Appendix for more details of the evolution of relative contribution of deformation mechanisms. 
(a)

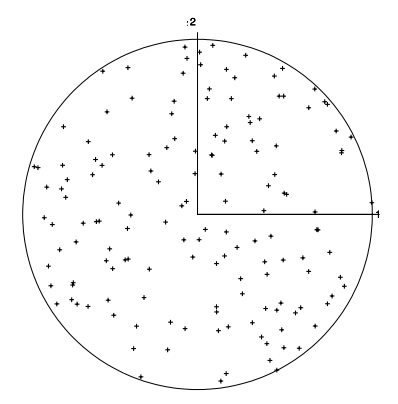

(c)

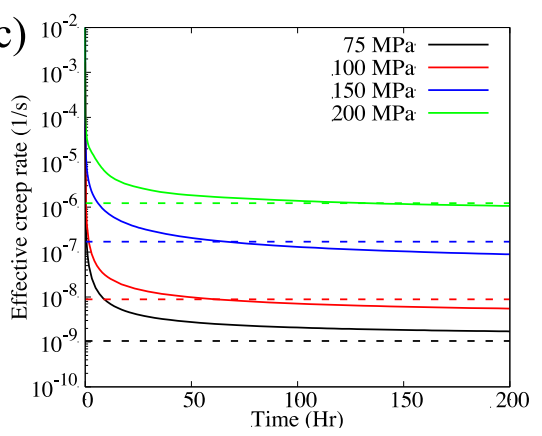

(b)

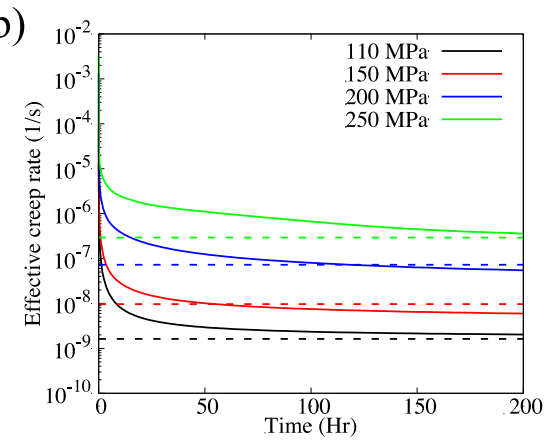

(d)

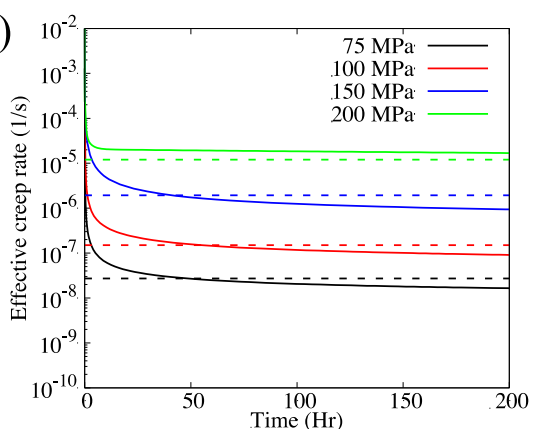

Fig. 1. (a) Pole figure for the initial random texture of 50 grains. VPSC model predicted thermal creep behavior of $316 \mathrm{H}$ steel as a function of stress at (b) $650{ }^{\circ} \mathrm{C}$, (c) $700^{\circ} \mathrm{C}$ and (d) $750{ }^{\circ} \mathrm{C}$. The dashed lines represent the experimentally measured steady state creep rate [65,66], whereas the solid lines are model predictions. Experimental results of $650^{\circ} \mathrm{C}$ and $110 \mathrm{MPa}, 650^{\circ} \mathrm{C}$ and $200 \mathrm{MPa}, 700^{\circ} \mathrm{C}$ and $100 \mathrm{MPa}, 750^{\circ} \mathrm{C}$ and $75 \mathrm{MPa}, 750^{\circ} \mathrm{C}$ and $100 \mathrm{MPa}$ cases were used for model calibration, and the remaining 7 cases are validated against the experiments.

\subsection{Material database and data reduction}

Polycrystal simulations are used to populate a database from which the SM will be derived. These simulations provide a significant amount of data (e.g. dislocation densities inside cells on each slip system and in each grain, etc.). Similarly to all viscoplastic constitutive models predicting material hardening or softening, the SM uses internal state variables (ISVs) to effectively quantify the materials state and history. The choice of the ISVs which can be extracted from polycrystal simulations is largely motivated by seminal work of Kocks and Mecking [25], in which macroscale 
flow stress is linked to dislocation density, taken as an effective value for the polycrystal. As such, homogenized dislocation densities are used to describe the evolving dislocation densities in the cells and cell walls contained in the crystal plasticity formulation. Two ISVs are included in the SM in this fashion, i.e.,

$$
\bar{\rho}_{\text {cell }}=\sum_{g}^{N_{g}} \sum_{s}^{N_{s}} \frac{\rho_{\text {cell }}^{g, s}}{N_{g} N_{s}}
$$

where $\rho_{\text {cell }}^{g, s}$ is the dislocation density in the cell of grain $g$ of $N_{g}$ grains, on slip system $s$ of $N_{s}$ slip systems, and $\bar{\rho}_{\text {cell }}$ is the homogenized dislocation density representing all cells in the polycrystal. A similar definition holds for the homogenized dislocation density within cell walls $\bar{\rho}_{W}$. Finally as per Kocks and Mecking [25] the accumulated Von Mises effective strain, $\bar{\varepsilon}_{v m}$, is used as a history tracking variable to account for strain hardening. Importantly, the material is assumed to be an annealed sample with random texture such that one expects the macroscale response of the material to be isotropic. Thus, rather than including individual tensor stress components, the Von Mises effective stress is recorded in the database and a Prandtl-Reuss flow rule is assumed. With this the database effectively tracks the evolution of $\bar{\varepsilon}_{v m}, \bar{\rho}_{W}, \bar{\rho}_{c e l l}, T$, and $\bar{\sigma}_{v m}$ following load increments.

A Prandtl-Reuss flow rule enforces an isotropic plastic response and ensures the homogenized behavior abides by the normality condition of Hill [66] and Rice [67]. Adherence to a maximal plastic work rate can be addressed in two parts: by enforcing an associated strain rate direction given a flow potential and by evaluating a scalar plastic multiplier proportional to the flow potential. With this the direction of plastic flow is determined by the deviatoric stress state. The magnitude of the plastic multiplier is estimated using interpolations between predicted values. 
Thus, while the homogenized data preserves the normality condition, the scalar rate of plastic flow may be subject to inaccuracies.

Given that the primary application of the SM will focus on predictions of creep response of the material, the database will be built from these types of simulations. Therefore, a fixed Von Mises stress and temperature are imposed and the evolution of $\bar{\varepsilon}_{v m}, \bar{\rho}_{W}, \bar{\rho}_{c e l l}$ are tracked as a function of time during each simulation. Information at each timestep is used to populate the database. Simulations are performed using different initial values of $\bar{\rho}_{W}, \bar{\rho}_{c e l l}, T$, and $\sigma_{v m}$ taken from within target ranges of those inputs. The target ranges are used to limit the collection of data. The evolution of $\bar{\varepsilon}_{v m}, \bar{\rho}_{W}$, and $\bar{\rho}_{c e l l}$ leads to data which are not contained by these target ranges. The surrogate model will operate on data normalized from the observation ranges. As such, the ranges used are larger than the target ranges to include all data within the normalized range. These are shown in Table 1.

Table 1 The ranges of the training and testing databases. The initial range corresponds to the values assigned at $t=0$ to the creep test simulations. The final range corresponds to the values obtained from considering each timestep of all the simulations

\begin{tabular}{|l|c|c|c|c|c|}
\hline & $\varepsilon_{v m}$ & $\begin{array}{c}\rho_{\text {cell }}\left(m^{-2}\right. \\
\left.\times 10^{12}\right)\end{array}$ & $\begin{array}{c}\rho_{W}\left(m^{-2}\right. \\
\left.\times 10^{12}\right)\end{array}$ & $\sigma_{v m}(\mathrm{MPa})$ & $T(\mathrm{~K})$ \\
\hline $\begin{array}{c}\text { Initial } \\
{[\text { min,max }]}\end{array}$ & {$[0,0]$} & {$[5,10]$} & {$[0.3,1]$} & {$[120,300]$} & {$[800,1000]$} \\
\hline $\begin{array}{c}\text { Final } \\
{[\text { min,max }]}\end{array}$ & {$[0,0.169]$} & {$[2.73,10]$} & {$[0.282,1]$} & {$[120,300]$} & {$[800,1000]$} \\
\hline
\end{tabular}

Importantly, it must be noted that with the choice of dead load creep simulations, the SM is trained only using monotonic loading conditions, and it is not known if evolution under complex loading can be addressed by the training data. Section 3 will therefore assess whether the SM can also be used under more complex loading. 
The dataset generated includes 9669 VPSC simulations for training and 2005 simulations for testing. The VPSC simulations are initialized with equal dislocation densities in all grains and slip systems. The densities $\left(\rho_{\text {cell }}\right.$ and $\left.\rho_{W}\right)$ used at $t=0$ and the temperature and applied $\sigma_{v m}$ are sampled from within the ranges shown in Table 1 labelled 'initial'. The simulations are run with 549 predetermined timesteps, for a total 58.5 hours. The timestep is initially small ( 1 second) and is increased incrementally as steady-state is approached. The SM uses the stress, temperature, and ISV values at timestep $i$ as inputs to predict the rates $\dot{\varepsilon}_{v m}, \dot{\bar{\rho}}_{c e l l}$, and $\dot{\bar{\rho}}_{w}$ at that timestep. Because the SM makes predictions in terms of individual time steps, the SM is trained on $9669 \times N_{t s}$ observations, where $N_{t s}=549$ is the number of timesteps in a simulation. Thus, more than 5.3 million training data are used.

The scheme with which the CP simulations are sampled within the domain (i.e. the design of computer experiments) must cover all areas within the target ranges without gaps. Space-filling designs $[68,69]$ minimize these gaps, but may introduce some bias due to regularity in spacing between samples. To avoid these issues, an approach giving similar results to orthogonal arraybased Latin hypercube sampling (LHS) [70] called LHS with multidimensional uniformity (LHSMDU) [71] is employed. This sampling design avoids the bias of a regularly-spaced grid design while still reducing the risk of large gaps in the database. Essentially, a random sampling is taken, from which points are removed to result in maximal distance between the remaining points. The strata selection in a LHS is used to match the rank ordering of the points within the first sample, in terms of each dimension. As a result, the average distance between points is slightly greater than for random sampling. An example with 5 strata is shown in the Appendix. The implementation of LHSMDU used here is obtained from published code [72]. To limit computation cost, the sampling scheme is used with an array of at most 100 strata (bins) in each 
dimension (e.g. 2 K per strata in T). Sample sets larger than 100 include multiple iterations of the LHSMDU procedure with different random seeds.

\section{$2.4 \quad$ Surrogate modeling of $\mathbf{3 1 6 H}$}

The mathematical form of a SM will necessarily impact computational cost and the trends which can be captured. The choice of model depends on consideration of two types of computational costs which are incurred in SM procedures. Training time costs take place as the mathematical form of the SM is made to fit the training database, and are incurred by once. Prediction time refers to the amount of operations involved in making use of the model. For the intended application of this work, it is critical to keep prediction time as small as possible.

Similarly to early efforts in response surface methodology which relied on polynomial regression models, the surrogate model uses polynomial regression [73] with Legendre polynomials as the orthogonal basis $[59,74]$. The choice of polynomial regression for this work allows data to be gathered as long as necessary, as no cost-scaling occurs as data increases. The orthogonal polynomials allow each term to have a solution which does not change if more terms are added. The surrogate formulation for $316 \mathrm{H}$ predicts rates from the input parameters using a regression modified by transformations on the inputs and outputs, i.e.,

$$
\begin{gathered}
\dot{\varepsilon}_{v m}^{* *} \sim \sum_{0 \leq i, j, k, l, m \leq N_{d e g}} \alpha_{\varepsilon}^{i j k l m} P^{i}\left(\bar{\rho}_{c e l l}^{*}\right) P^{j}\left(\bar{\rho}_{w}^{*}\right) P^{k}\left(T^{*}\right) P^{l}\left(\sigma_{v m}^{*}\right) P^{m}\left(\varepsilon_{v m}^{*}\right) \\
\dot{\bar{\rho}}_{c e l l}^{* *} \sim \sum_{0 \leq i, j, k, l, m \leq N_{d e g}} \alpha_{\rho_{c e l l}}^{i j k l m} P^{i}\left(\bar{\rho}_{c e l l}^{*}\right) P^{j}\left(\bar{\rho}_{w}^{*}\right) P^{k}\left(T^{*}\right) P^{l}\left(\sigma_{v m}^{*}\right) P^{m}\left(\varepsilon_{v m}^{*}\right) \\
\dot{\bar{\rho}}_{w}^{* *} \sim \sum_{0 \leq i, j, k, l, m \leq N_{d e g}} \alpha_{\rho_{w}^{i j k l m}} P^{i}\left(\bar{\rho}_{c e l l}^{*}\right) P^{j}\left(\bar{\rho}_{w}^{*}\right) P^{k}\left(T^{*}\right) P^{l}\left(\sigma_{v m}^{*}\right) P^{m}\left(\varepsilon_{v m}^{*}\right)
\end{gathered}
$$


where each $i, j, k, l$, and $m$ is an index corresponding to the degree of the Legendre polynomial, $P^{i}$, generated from the transformed input parameter (e.g. $T^{*}$ ) indicated in the subscript. The summation occurs over all five inputs. The coefficients are specific to the output as is denoted in superscript. With functional forms so defined, the SM can be fit to VPSC data.

The reduced input parameter basis, $\bar{\varepsilon}_{v m}, \bar{\rho}_{W}, \bar{\rho}_{c e l l}, T$, and $\sigma_{v m}$, and the outputs $\dot{\varepsilon}_{v m}, \dot{\bar{\rho}}_{c e l l}$, and $\dot{\bar{\rho}}_{W}$ are transformed prior to the polynomial regression. The transformations on the inputs improve SM performance when non-polynomial (e.g. exponential) dependence is present. The output transformations force the solutions of the polynomial to mimic physically observed properties of the outputs. For example for $\dot{\rho}_{\text {cell }}$, a transformation of $\dot{\rho}_{\text {cell }}^{* *}=\ln \left(\frac{-\dot{\rho}_{\text {cell }}}{\rho_{\text {cell }}}\right)$ prevents evolution to negative densities and allows the SM to better fit evolution rates of different orders of magnitude. To allow for support of negative and positive rates, a piecewise mapping is used, i.e.,

$$
y=\left\{\begin{array}{c}
\ln (z+m) \quad \text { if } z>0 \\
\ln \left(-\frac{m}{\frac{z}{m}-1}\right) \text { if } z \leq 0
\end{array}\right.
$$

where $\mathrm{y}$ is the $\mathrm{SM}$-native output, $\mathrm{z}$ is the engineering output, and $m$ is a constant used to tune the mapping. This piecewise mapping is used with multiple variables. A full list of the input and output mappings which are used in this application are shown in Table 2. In Table 2, the piecewise mapping is denoted as $p w(z, m)$. These mappings are applied prior to normalizing to the interval $[-1,1]$ for generating Legendre polynomial terms. Outside this interval, the polynomials are not orthogonal. The normalization makes the training interval coincident with the orthogonal interval. 
Table 2 The mappings from engineering units to SM transformed units in the demonstration model

\begin{tabular}{|c|r|c|c|c|c|c|}
\hline \multicolumn{2}{|c|}{ Inputs } & $\varepsilon_{v m}^{*}$ & $\rho_{\text {cell }}^{*}\left(m^{-2}\right)$ & $\rho_{W}^{*}\left(m^{-2}\right)$ & $\begin{array}{c}\sigma_{v m}^{*} \\
(\mathrm{MPa})\end{array}$ & $T^{*}(\mathrm{~K})$ \\
\hline$\dot{\varepsilon}_{v m}^{* *}$ & $\ln \left(\dot{\varepsilon}_{v m}\right)$ & $\begin{array}{c}\ln \left(\varepsilon_{v m}\right. \\
\left.+10^{-4}\right)\end{array}$ & $\rho_{\text {cell }}$ & $\ln \left(\rho_{W}+1\right)$ & $\begin{array}{l}\ln \left(\sigma_{v m}\right. \\
+5)\end{array}$ & $T$ \\
\hline$\dot{\bar{\rho}}_{\text {cell }}^{* *}$ & $p w\left(-\frac{\dot{\rho}_{\text {cell }}}{\rho_{\text {cell }}}, 10^{-10}\right)$ & $\begin{array}{c}\ln \left(\varepsilon_{v m}+\right. \\
\left.4 \times 10^{-5}\right)\end{array}$ & $\ln \left(\rho_{\text {cell }}+1\right)$ & $\ln \left(\rho_{W}+1\right)$ & $\begin{array}{c}\ln \left(\sigma_{v m}\right. \\
+10)\end{array}$ & $T$ \\
\hline$\dot{\bar{\rho}}_{w}^{* *}$ & $p w\left(\frac{\dot{\rho}_{W}}{\rho_{W}}, 2 \times 10^{-10}\right)$ & $\varepsilon_{v m}$ & $\exp \left(\frac{\rho_{\text {cell }}}{1 \times 10^{13}}\right)$ & $\ln \left(\rho_{W}+1\right)$ & $\begin{array}{c}\ln \left(\sigma_{v m}\right. \\
+10)\end{array}$ & $\begin{array}{c}\ln (T \\
+1)\end{array}$ \\
\hline
\end{tabular}

Fitting the SM to data is a two-step procedure, involving both training and testing. The coefficients $\alpha$ are evaluated, or "trained", by fitting each regression model to data. Training is accompanied by testing, where the trained model is applied to additional testing data, and the trained model's testing predictions are "tested" against corresponding "true" testing values.

Testing data is compared to the trained regression model predictions to provide an indication of performance, via the $R_{\text {testing }}^{2}$ value of the trained predictions on the test data. This $R_{\text {testing }}^{2}$ value is calculated as,

$$
R_{\text {testing }}^{2}=1-\frac{\sum_{\text {testing }}\left(y_{S M}-y_{C P}\right)^{2}}{\sum_{\text {testing }}\left(y_{S M}-\bar{y}_{C P}\right)^{2}}
$$

where $y_{S M}$ is a model prediction corresponding to a true testing data point $y_{C P}$, and $\bar{y}_{C P}$ is the mean value of the output $y$ across all of the testing data. This $R_{\text {testing }}^{2}$ value is used to select the optimal value to use for the maximum degree, $N_{\text {deg }}$; if there is overfitting (which occurs when the regression model is able to fit to noise in the data), $R_{\text {testing }}^{2}$ scores will decrease (even as training performance continues to increase). For the $316 \mathrm{H} \mathrm{SM}$, testing performance is best using a maximum degree of 3 . 


\section{Results and Discussion}

\subsection{Model validation in dead load creep scenarios}

In a first application, creep test simulations are used to evaluate the fidelity of the surrogate models across the targeted range of applied stresses, temperatures, and initial ISV values. The same ranges are used for the validation simulations as for the training and testing simulations. A separate LHSMDU-based sampling is used to generate the 301 validation simulations.

Importantly, whereas the training data and testing data are provided to the SM as individual time-steps, the validation performance is evaluated for each simulation, i.e.,

$$
R_{\text {validation }}^{2}=1-\frac{\sum_{i}\left(\varepsilon_{v m, i}^{S M}-\varepsilon_{v m, i}^{V P S C}\right)^{2}}{\sum_{i}\left(\varepsilon_{v m, i}^{V P S C}-\bar{\varepsilon}_{v m}^{V P S C}\right)^{2}}
$$

where $i$ refers to the $i$-th timestep in the simulation and $\bar{\varepsilon}_{v m}^{V P S C}$ is the average of the strain values over all the timesteps in the simulation.

$R_{\text {validation }}^{2}$ is measured in terms of either creep strain or an ISV (i.e. $\bar{\rho}_{W}$ or $\bar{\rho}_{c e l l}$ ), and the closer it is to 1 the better the performance of the SM is. To present data in greater than 3 dimensions, a scatterplot matrix is employed. For example, the $R_{\text {validation }}^{2}$ in terms of the creep strain is shown in Fig. 4, with each validation run evaluated by comparing each timestep in the SM emulation to the VPSC equivalent. In the scatterplot matrix, each pairwise combination of the target dimensions is included, using common axes to align the combinations in a gridded format. The corresponding value of $R_{\text {validation }}^{2}$ informs the color of the simulation markers. For the vast majority of the target domain, the VPSC simulation is emulated at high fidelity. Of all 301 validation cases, only 6 showed fits to VPSC with $\mathrm{R}^{2}$ values at or below 0.95 for $\varepsilon_{v m}$, which is the value of most concern to applications. 


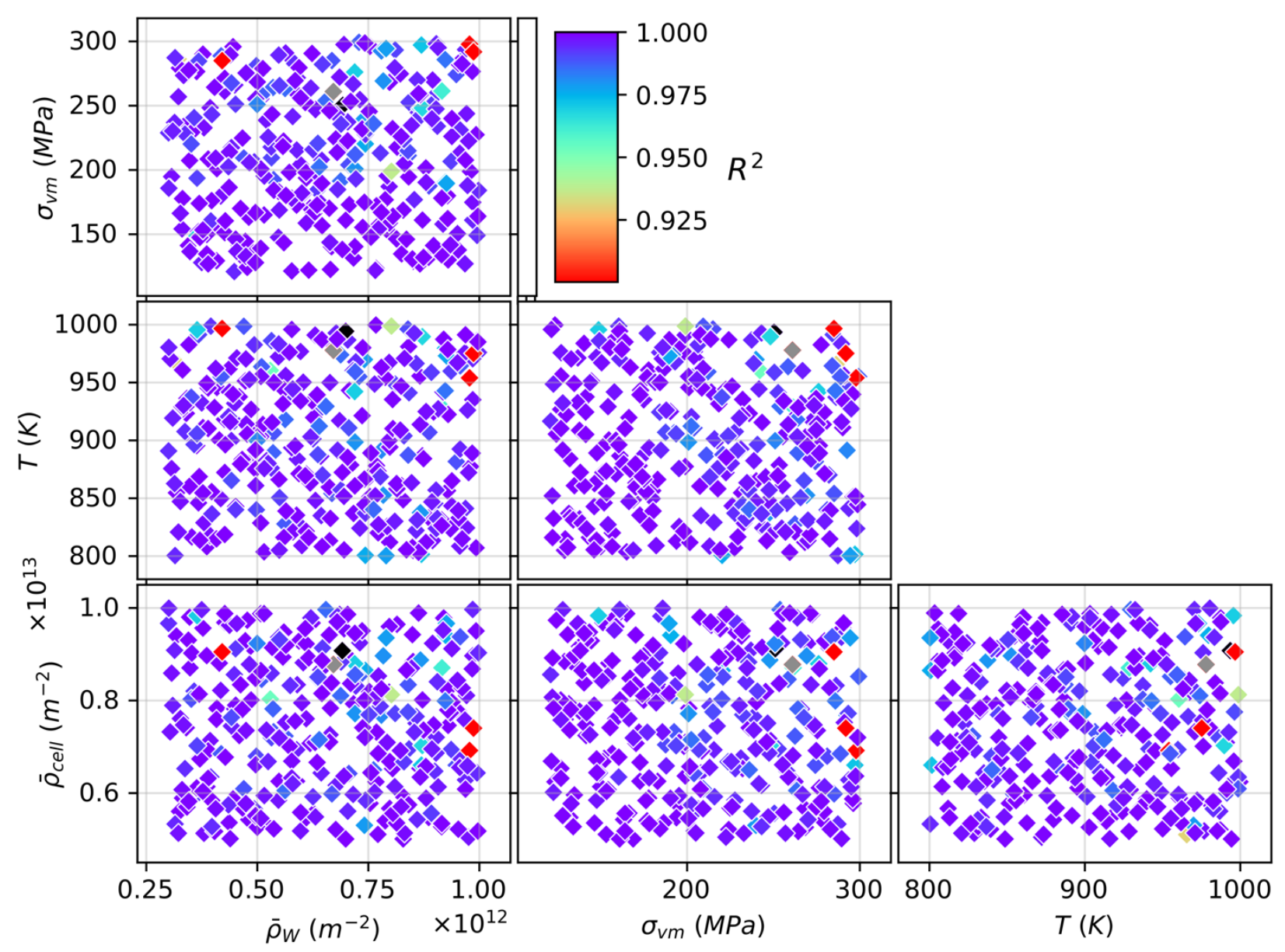

Fig. 2 Validation of $S M$ in-terms of $R_{v a l i d a t i o n}^{2}$ value of the $\varepsilon_{v m}$ prediction. The database of validation cases is presented using a scatterplot matrix, each pairwise combination of the target dimensions is included as a subplot, using common axes to align the combinations in a gridded format. Each case appears once in each subplot. Two cases are highlighted in black and grey, respectively, with $R_{\text {validation }}^{2}$ equal to 0.9977 and 0.844 . Note that out of all 301 validation cases, only 6 cases present $R_{\text {validation }}^{2}$ values less than 0.95

For the sake of clarity, two predictions of the creep load response of the material are shown explicitly. The initial conditions associated with these are shown in Fig. 2 as the black point $\left(R_{\text {validation }}^{2}=0.9977\right)$ and the grey point $\left(R_{\text {validation }}^{2}=0.844\right)$. For the case with $R_{\text {validation }}^{2}$ equal to 0.9977 , the SM predictions are shown in the upper half of Fig. 3. The three isotropic outputs, $\bar{\rho}_{c e l l}, \bar{\rho}_{w}$, and $\varepsilon_{v m}$, are shown in 3(a), 3(b), and 3(c), respectively. The SM predictions are 
shown in blue, alongside the values calculated from the VPSC simulation in red. As shown, the fit of the SM to the VPSC results is excellent as it is difficult to distinguish the two series.

a)

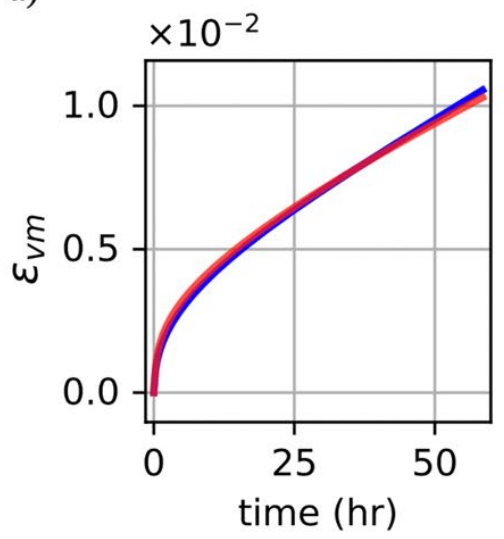

d)

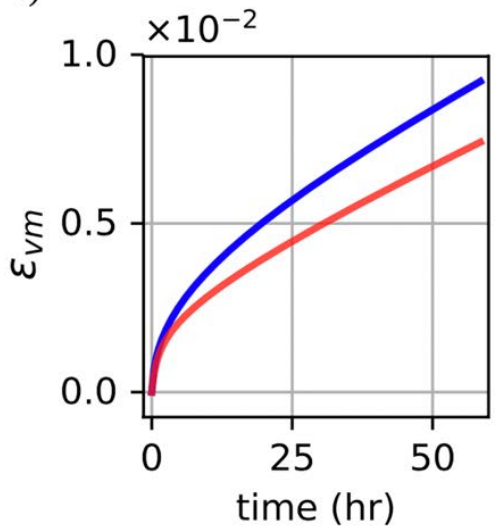

b)

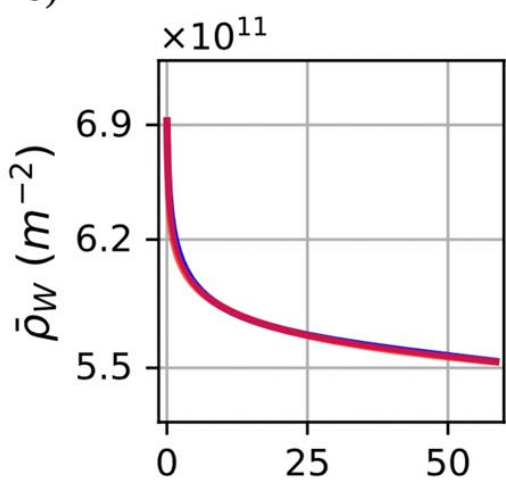

e)

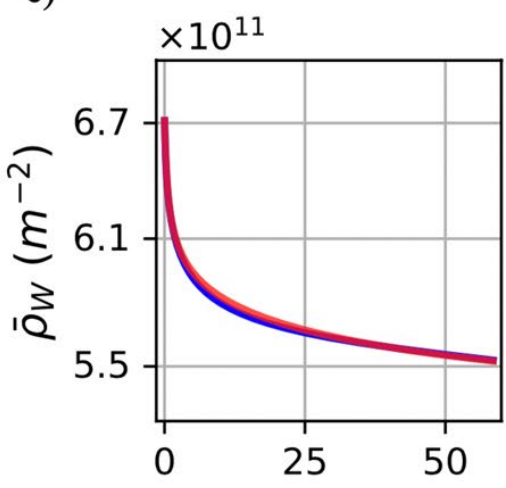

c)

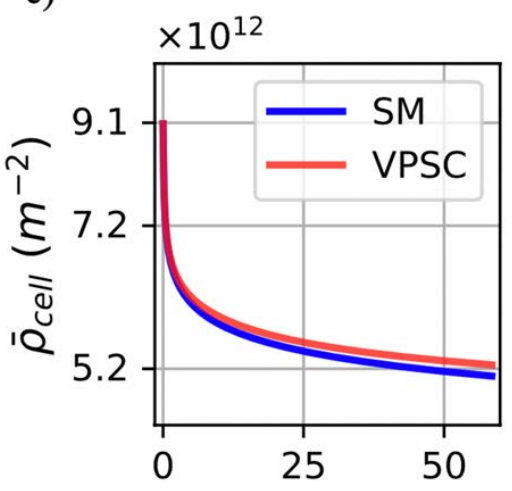

f)

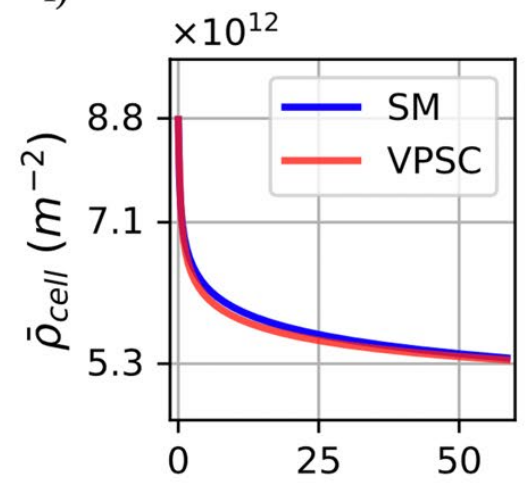

Fig. 3 Two cases of SM and VPSC predicted evolution of creep strain, dislocation density in the cell and in the cell-wall. Above, the simulation corresponds to initial values of $\sigma_{v m}=250.3 \mathrm{MPa}$, $T=993.5 K, \rho_{\text {cell }}=9.1 \times 10^{12} \mathrm{~m}^{-2}$ and $\rho_{\text {wall }}=6.9 \times 10^{11} \mathrm{~m}^{-2}$. Below, the simulation corresponds to $\sigma_{v m}=261 \mathrm{MPa}, \mathrm{T}=977.9 \mathrm{~K}, \rho_{\text {cell }}=8.779 \times 10^{12} \mathrm{~m}^{-2}$ and $\rho_{\text {wall }}=6.714 \times$ $10^{11} \mathrm{~m}^{-2}$. The $R^{2}$ value of the lower case $\varepsilon_{v m}$ prediction compared to the VPSC simulation is 0.844

The individual SM predictions for the lowest $R_{\text {validation }}^{2}$ case are shown in the lower half of Fig. 3. The evolution of $\varepsilon_{v m}, \bar{\rho}_{c e l l}$, and $\bar{\rho}_{w}$ is shown as a function of time in Fig. 3(d), 3(e), and 3(f), respectively, with the SM shown in blue and the CP shown in red. The SM underpredicts 
strain accumulation by around $15 \%$ of the VPSC result. Additionally, the timing of the transition from the initial creep strain rate to the steady-state creep rate is consistent between the SM and the CP-VPSC.

As the surrogate model aims to be integrated within FEM solvers, it is critical to assess how the use of Von Mises stresses (as opposed to using the full stress matrix) affects the predictions of all plastic strain components in the system. A simulation case is selected, with input values $\sigma_{v m}=276.6 \mathrm{MPa}, T=889 \mathrm{~K}, \rho_{\text {cell }}=8.683 \times 10^{12} \mathrm{~m}^{-2}$, and $\rho_{\text {wall }}=9.84 \times$ $10^{11} \mathrm{~m}^{-2}$. The $\mathrm{J} 2$ plasticity assumption is used to reconstitute the full strain rate tensor, i.e.,

$$
\dot{\varepsilon}_{i j}=\frac{3}{2} \sigma_{i j} \frac{\dot{\varepsilon}_{v m}}{\sigma_{v m}}
$$

from the SM prediction of effective strain rate, $\dot{\varepsilon}_{v m}$, where $\sigma_{i j}$ is the deviatoric stress tensor. This calculated value is compared against the VPSC results, shown in Fig. 4. This strain rate tensor is needed in applications of the SM to FEM simulations. With the same color scheme as Fig. 3, the axial strain components and the shear strain components are compared in the left and right-hand columns, respectively. The VPSC strain components are calculated directly, whereas the SM strain components are calculated using the SM-predicted effective strain rate, given by Eq. (11). The comparison shows a good match between the strain tensor from VPSC and the J2 approximation used to generate the strain tensor components from the SM predictions. This gives support for the accuracy maintained with the $\mathrm{J} 2$ simplification, with which a reduction of floating-point operations of at least 243 times is obtained versus a SM without the simplification. 

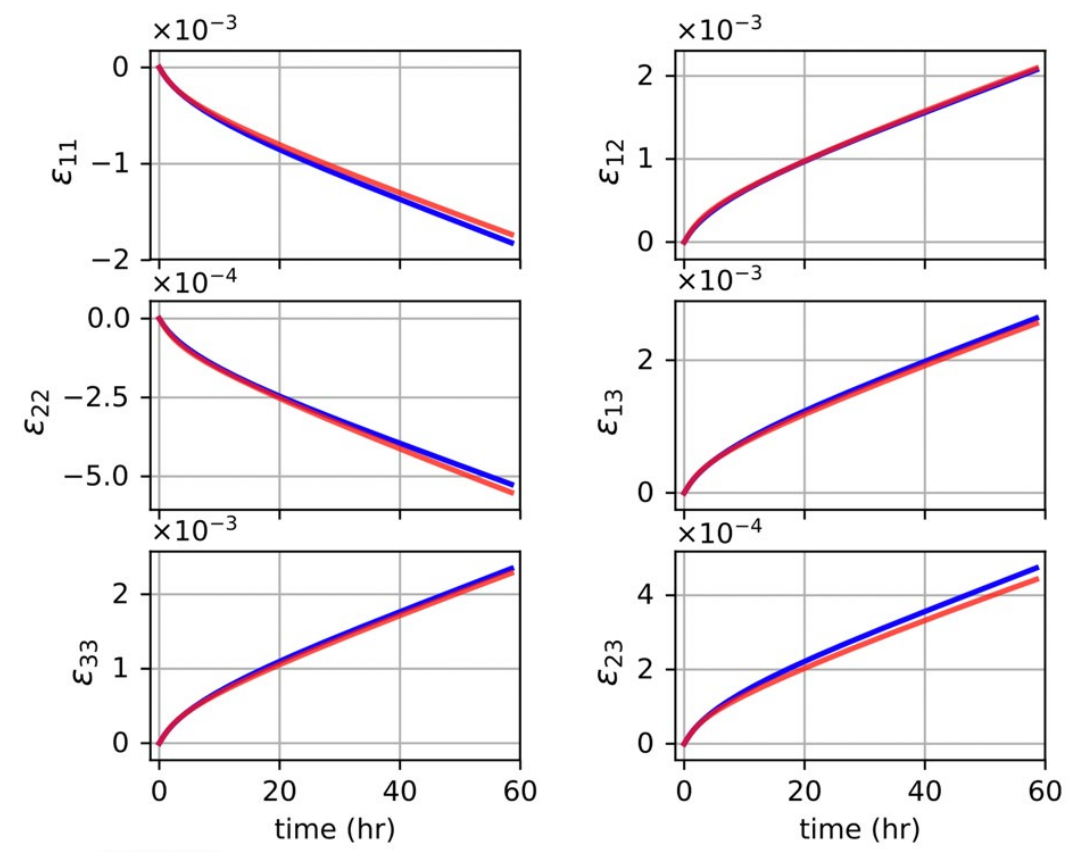

Fig. 4 SM (blue) and VPSC (red) predicted evolution of creep strain in terms of the components of the strain tensor. The SM series are recovered from the isotropic rate prediction using a J2 flow potential. This simulation corresponds to $\sigma_{v m}=276.6 \mathrm{MPa}, \mathrm{T}=889 \mathrm{~K}, \rho_{\text {cell }}=8.683 \times$ $10^{12} \mathrm{~m}^{-2}$ and $\rho_{\text {wall }}=9.84 \times 10^{11} \mathrm{~m}^{-2}$. The $\mathrm{J} 2$ simplification results in minimal departure from the precise stress tensor obtained with VPSC

The speed-up obtained by the SM relative to the VPSC simulation varied from a factor of 10 to more than 100. This is as expected, as the VPSC model converges at different rates for different stresses and temperatures while the cost of the SM is independent of the boundary conditions and microstructure state.

\subsection{Complex Loading Histories}

The developed SM is employed to simulate complex non-monotonic loading histories. None of the following tests are used to inform or train the SM. We consider three different loading histories: (i) temporary load increase; (ii) creep under cyclic stress; and (iii) creep under thermal cycling. 
First, to test whether the developed SM can capture the effect of temporary load increase on creep transients, a simulation is run with a fixed load of $134.4 \mathrm{MPa}$ for 18.22 hours, an increased load of 268.8 MPa for 2.25 hours, followed by another 51.14 hours at $134.4 \mathrm{MPa}$. Fig. 5(a) shows the schematic representation of imposed load history. The predicted evolutions of creep strain, and dislocation densities in the cell and in the cell-wall are shown in Fig. 5(b-d). The SM and VPSC results are shown in blue and red, respectively. The agreement between SM and VPSC predictions is good $R^{2}=0.986$. Both models capture the sudden increase in the creep strain-rate and abrupt changes in the ISV rates due to elevated loading. To the extent that CP-VPSC modeling can capture the transients induced by a period of elevated loading, the SM can as well. 

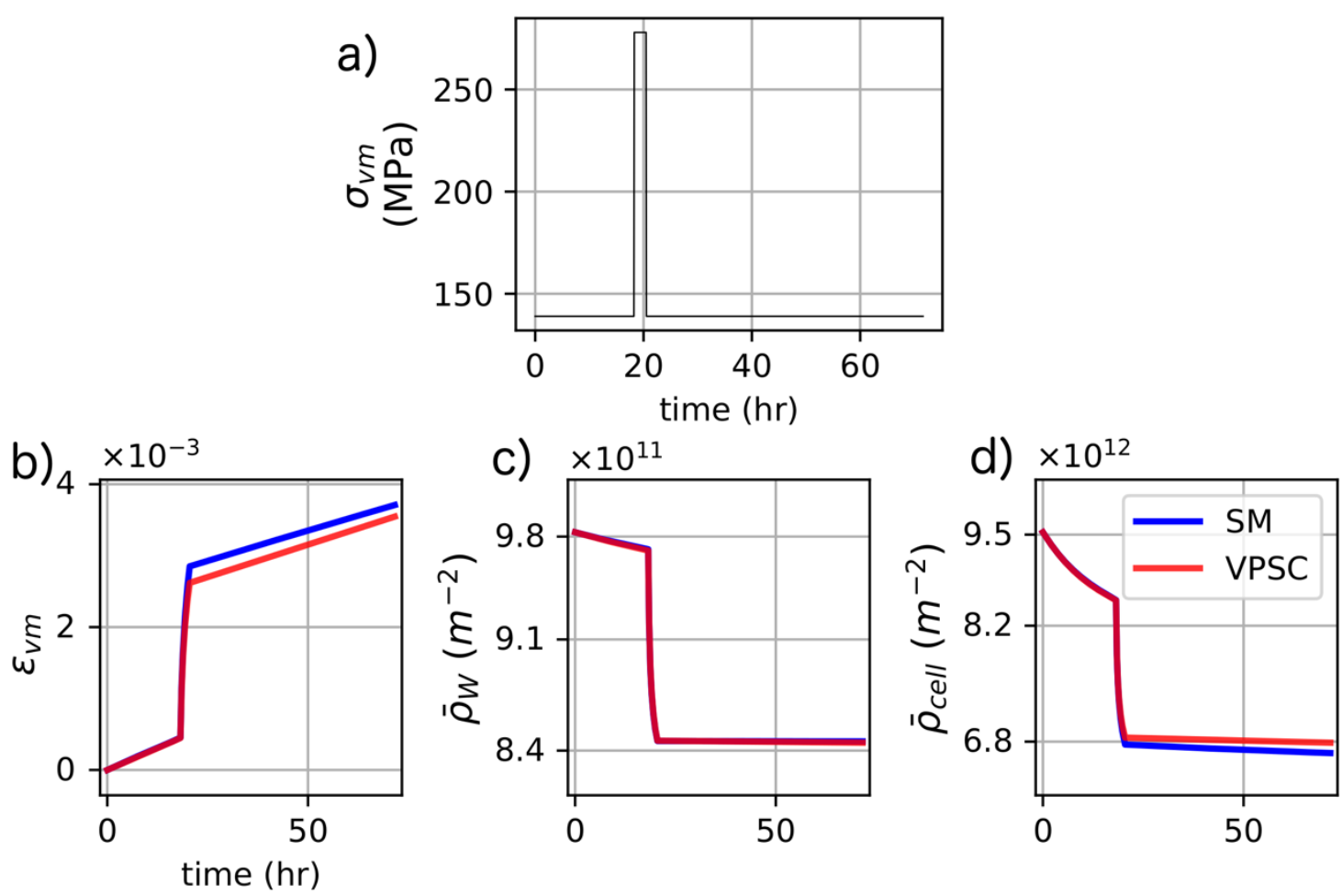

Fig. 5 Validation of SM prediction for creep test with temporary elevated loading. a) The imposed stress profile which after 18.22 hours suddenly increases the stress from $134.4 \mathrm{MPa}$ to $268.8 \mathrm{MPa}$ for 2.25 hours. Other simulation conditions are: $T=910.6 K, \rho_{\text {cell }}=8.758 \times$ $10^{12} \mathrm{~m}^{-2}$, and $\rho_{\text {wall }}=6.871 \times 10^{11} \mathrm{~m}^{-2}$. SM and VPSC predicted the evolution of b) creep strain, c) dislocation density in the cell, and d) cell-wall. SM correctly captures the impulsive loading induced transients

Second, the response of the SM to creep fatigue loading is investigated. Three cases are chosen with different mean stress values, $\sigma_{m}=150,210$ and $270 \mathrm{MPa}$, and the same stress amplitude, $\sigma_{a}=30 \mathrm{MPa}$. The stresses and temperatures were chosen from a region of the validation ranges for which the SM exhibits good performance. Schematic representation of the imposed stress profile is shown in Fig. 6(a). The three simulations are compared to the results of VPSC simulations under the same conditions, as shown in Fig. 6(b). The SM and VPSC results are closely matched, much more so at the lower mean stresses. At higher stress, the values of 
$\bar{\varepsilon}_{v m}, \bar{\rho}_{W}$, and $\bar{\rho}_{c e l l}$ evolve more rapidly. Because the database is obtained from monotonic load simulations, the evolution of $\bar{\varepsilon}_{v m}, \bar{\rho}_{W}$, and $\bar{\rho}_{\text {cell }}$ in these cycling tests deviates from the bulk of the database. Due to this, the 270 MPa SM simulation is less accurate as evolution takes place, specifically above a $\bar{\varepsilon}_{v m}$ value of $2 \times 10^{-3}$.

a)

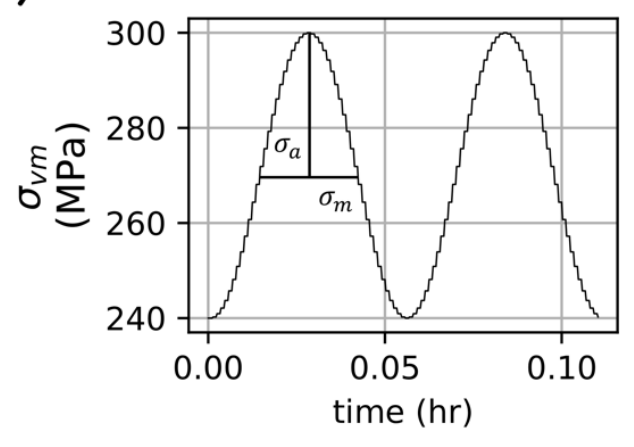

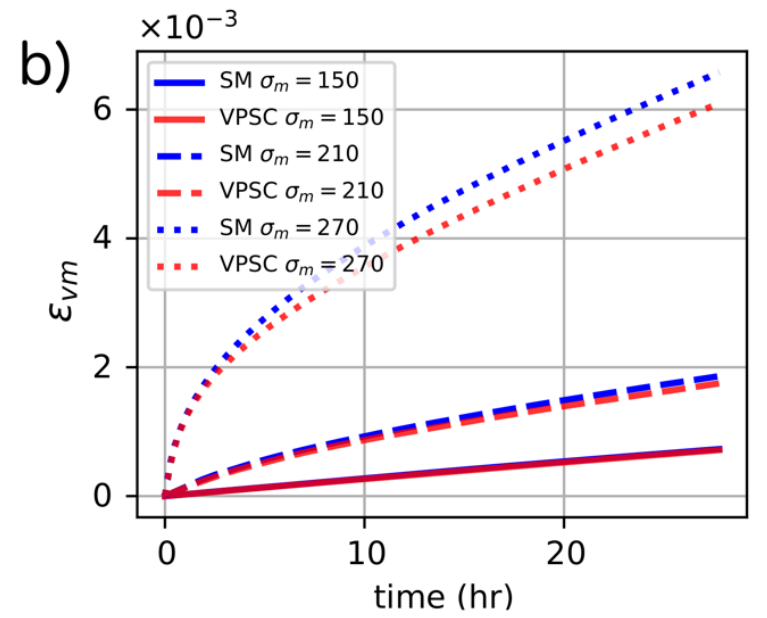

Fig. 6 Prediction of low cycle fatigue creep using SM and VPSC for a) cyclic loading condition, using input values for which the SM provides good results in dead load creep. Here $\sigma_{m}$ and $\sigma_{a}$ refer to mean stress and stress amplitude. For all cases, we fix the stress amplitude as $30 \mathrm{MPa}$. Other simulation conditions are: $T=973.0 \mathrm{~K}, \rho_{\text {cell }}=7.0 \times 10^{12} \mathrm{~m}^{-2}$ and $\rho_{\text {wall }}=7.0 \times$ $10^{11} \mathrm{~m}^{-2}$. b) SM and VPSC predicted creep strain evolution for 500 cycles with $\sigma_{m}=$ 150,210 and 270 MPa. SM correctly captures the effect of load cycle on the creep strain

Finally, the SM can respond to a fluctuation in temperature within a given simulation. To demonstrate this capability, a simulation of creep under thermal cycling is shown in Fig. 8. A single five-hour thermal cycle is shown in Fig. 7(a), where temperature is held at $903 \mathrm{~K}$ and 803 $\mathrm{K}$ with constant heating and cooling rates in transitions. The applied stress is $250 \mathrm{MPa}$ for all cases. The comparison is made between the SM and CP-VPSC. Creep strain is shown as a function of time in Fig. 7(b). In addition to the cyclic temperature simulations which are shown in black (CP) and red (SM), constant $\mathrm{T}$ cases are shown in dotted lines for $\mathrm{CP}$ and solid lines for SM, at $803 \mathrm{~K}$ 
in blue and $903 \mathrm{~K}$ in green. The SM matches the CP simulations closely in all cases. It can be seen that the thermal cycling leads to strain accumulations which fall between the constant temperature simulations. In Fig. 7(c), the creep strain rates of these simulations are shown. The approximate nature of the SM is more apparent in the plot of creep strain rate, as is discussed in Section 3.3. The inaccuracies in the SM predictions are consistent between the constant temperature cases and the thermal cycling case. It can be seen in Fig. 7(c) that the presence of thermal cycling can lead to higher instantaneous creep rates than constant high temperature creep tests. This is expected, as this difference is a result of slower saturation of dislocations in the cycling case.
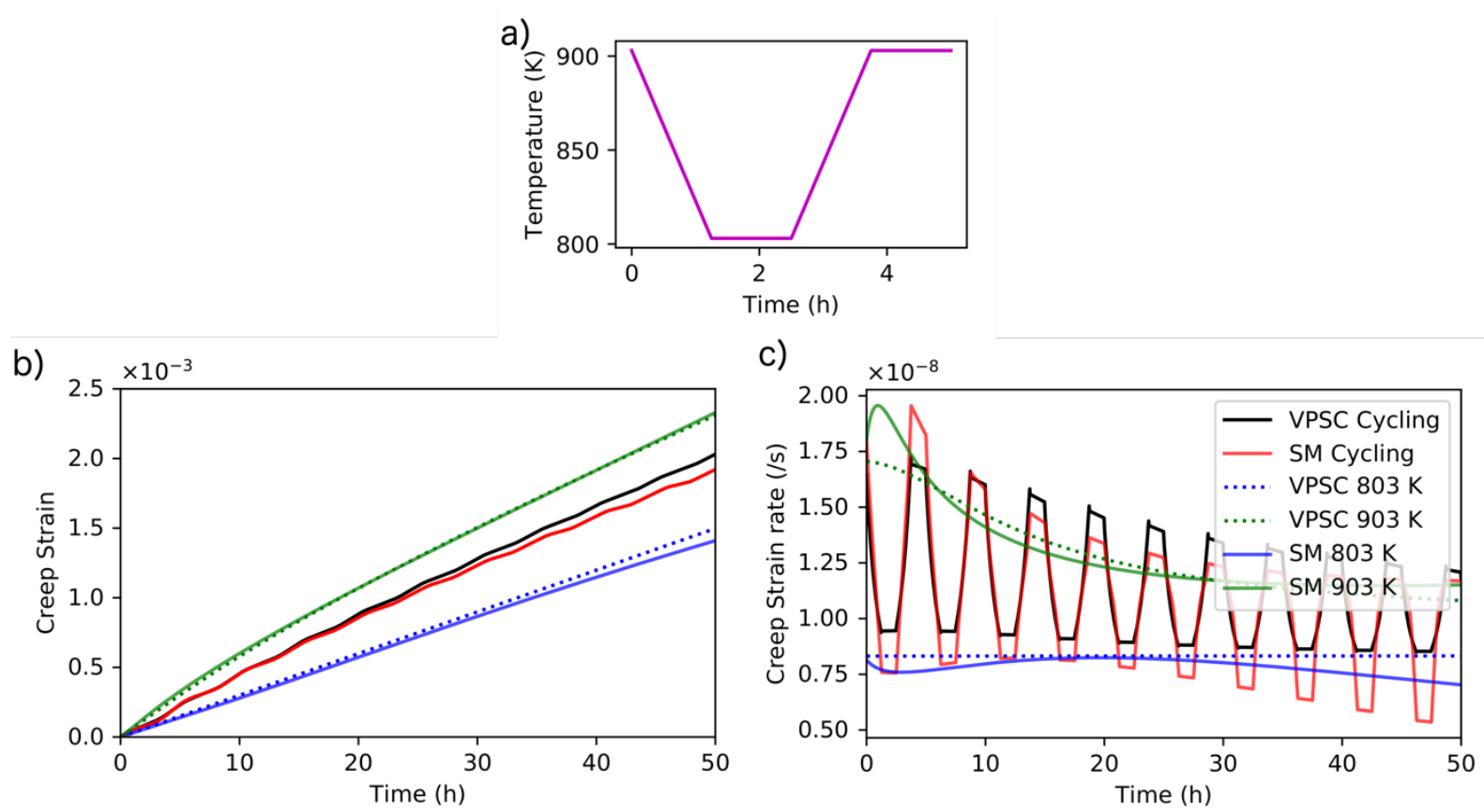

Fig. 7 Prediction of creep behavior under the thermal cycle shown in a). The simulation is performed for 10 cycles. Other simulation conditions are: $\sigma=250.0 \mathrm{MPa}, \rho_{\text {cell }}=6.0 \times$ $10^{12} \mathrm{~m}^{-2}$ and $\rho_{\text {wall }}=6.0 \times 10^{11} \mathrm{~m}^{-2}$. SM and VPSC predicted b) creep strain and c) creep rate behaviors for fixed temperature 


\subsection{Surrogate Modeling of Strain Rate}

The SM predictions of accumulated strain are consistently accurate. However, the strain rate predictions highlight some limitations of the SM as currently implemented. The mean squared relative error (MSRE) of the strain rate, i.e.,

$$
\operatorname{MSRE}=\frac{1}{N_{\text {timesteps }}} \sum_{i}^{N_{\text {timesteps }}}\left(\frac{\dot{\varepsilon}_{v m, i}^{S M}-\dot{\varepsilon}_{v m, i}^{V P S C}}{\dot{\varepsilon}_{v m, i}^{V P S C}}\right)^{2}
$$

in the validation set of creep simulations is shown in Fig. 8. A value of MSRE above 1 indicates that the predictions are quite inaccurate, with errors of $100 \%$ or more. This can occur even when the accumulated strain is predicted accurately, as the strain rate errors can cancel out over the course of a creep simulation. 


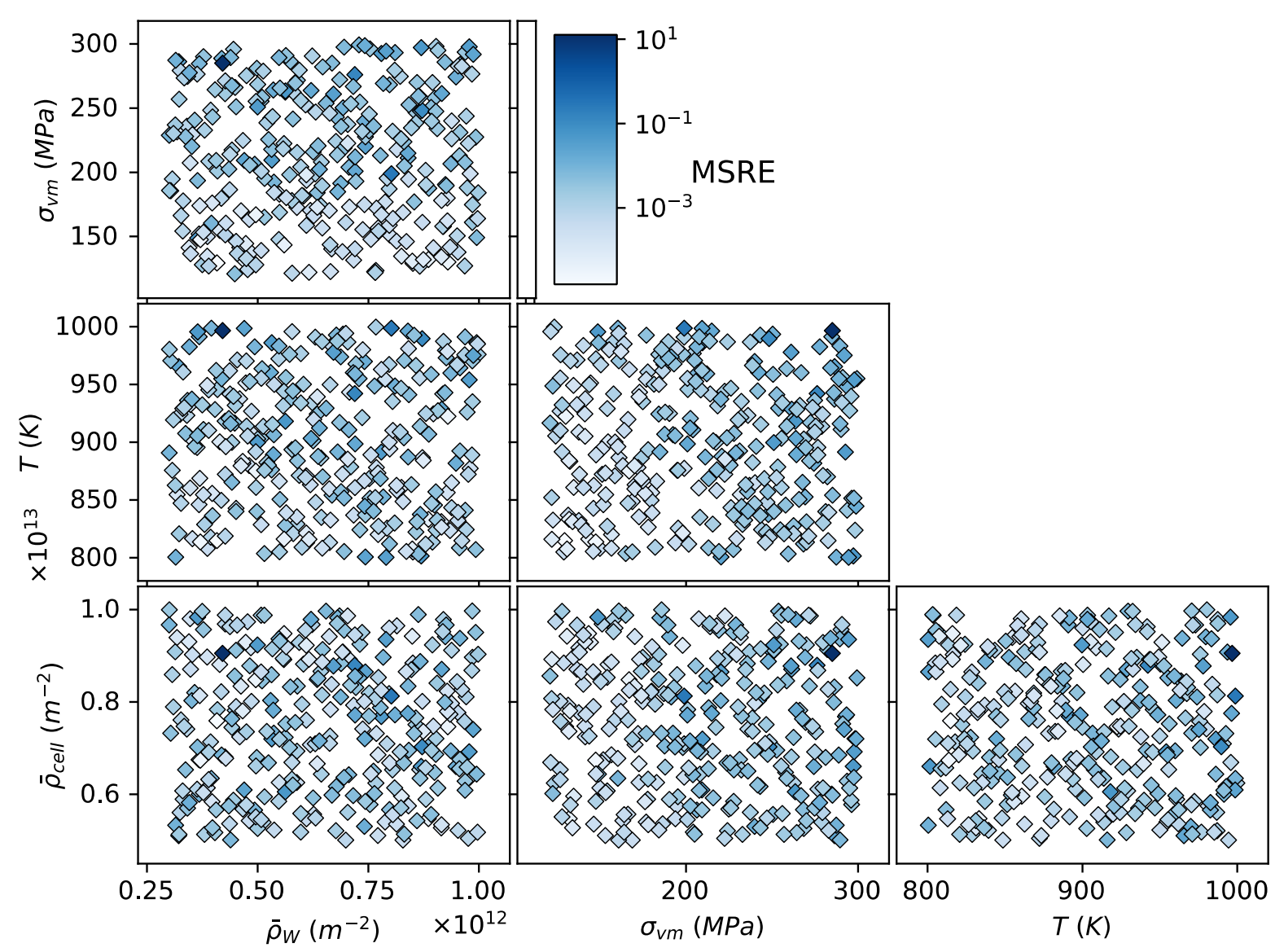

Fig. 8 The MSRE of $\dot{\varepsilon}_{v m}$ for the dead load creep simulations used in validation. The database of validation cases is presented using a scatterplot matrix, each pairwise combination of the target dimensions is included as a subplot, using common axes to align the combinations in a gridded format. Each case appears once in each subplot

The SM uses the input and output mappings to approximate physics-based model responses, even when the response is not suited to a polynomial fit. For example, the SM fits the stress dependence of power law creep. A polynomial cannot approximate a power law, however the input mapping allows the polynomial to fit the response as if it was linear. When two or more mechanisms trade dominance over the target range of stress, one mapping is insufficient. The mapping which renders power law stress dependence linear will make the dependence of diffusion creep non-linear, and vice-versa. Polynomial fits of these non-linear trends can result in artifacts, 
and the errors from these artifacts will typically cancel out somewhat when an average is taken. Given that the proportion of Coble creep predicted by the VPSC simulations varied from 0 to $100 \%$ within the target ranges, these issues are likely the primary cause of the observed strain rate errors. The mapping issue can be addressed by applying the SM to smaller subregions. If there is less variation in the dominant mechanism, less non-linearity will be left in the mapped responses. This approach is shown in Fig. 9. Two subdomains were chosen: one at higher stresses $\left(\sigma_{v m}=[200,300] \mathrm{MPa}, T=[800,950] \mathrm{K}\right)$ and one at lower stresses $\left(\sigma_{v m}=[120,150] \mathrm{MPa}\right.$, $T=[800,1000] \mathrm{K})$. Additional simulations were collected (1682) to supplement the existing data for each subdomain, for totals of 5681 and 3341 simulations, respectively. New input mappings were identified for each subdomain to optimize a linear fit and are shown in Table 3. Output mappings were not adjusted. Fig. 9(a) and 9(d) show the MRSE values for the original validation cases using the subdomain SMs for high and low stresses, respectively. The worst cases of the subdomain SM are as accurate as the best cases of the original SM. A comparison of $\dot{\varepsilon}_{v m}$ predictions is shown in detail in Fig. 9(b) and 9(c) for the high stress SM and in Fig. 9(e) and 9(f) for the low stress SM. The SM results are shown in blue and the VPSC results are shown red. Fig. 9(c) and 9(f) are the original SM predictions of $\dot{\varepsilon}_{v m}$, and Fig. 9(b) and 9(e) show the new subdomain SM predictions. 

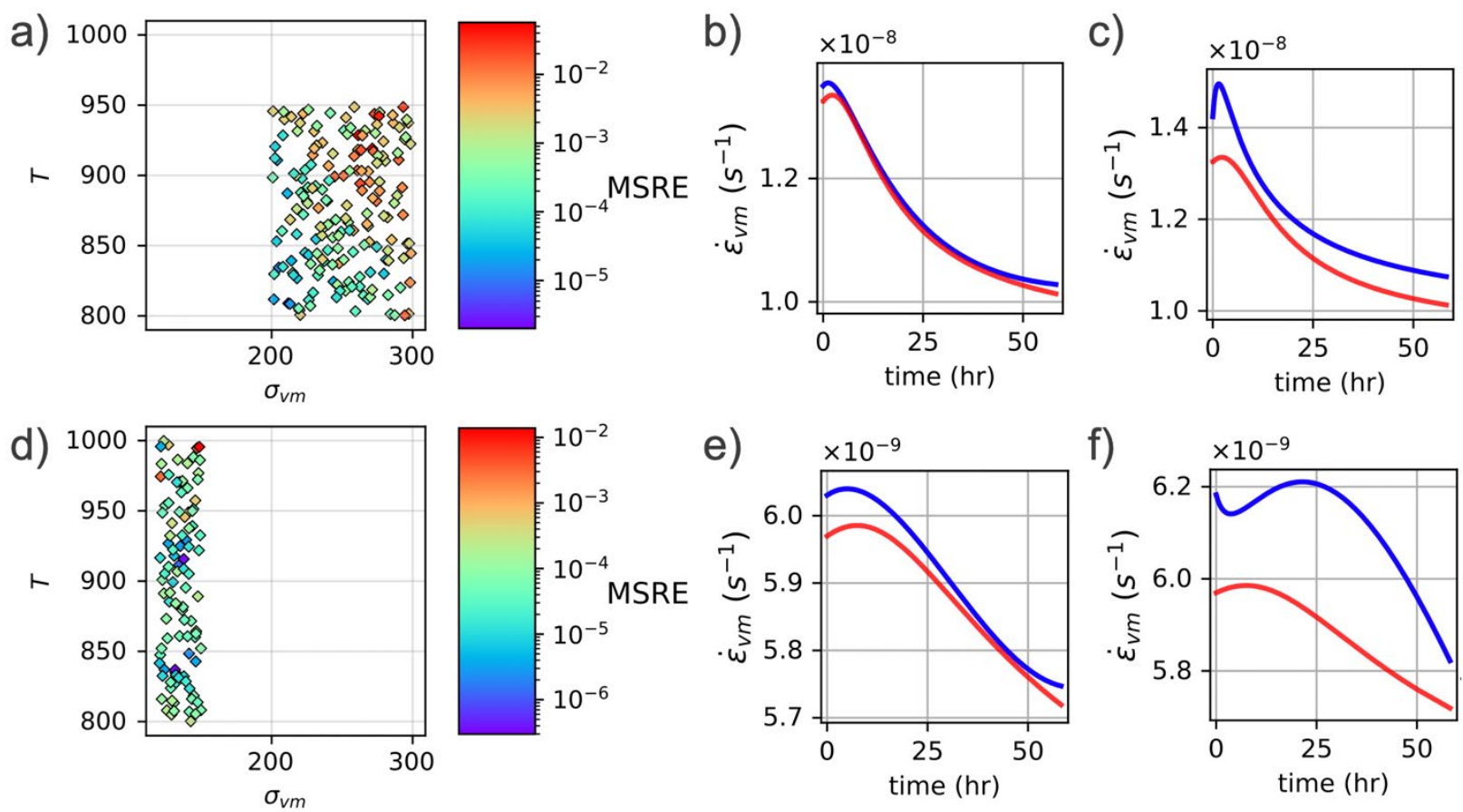

Fig. 9 The results of the subdomain SMs in validation. (a), (b), and (c) correspond to the high stress subdomain and (d), (e), and (f) correspond to the low stress subdomain. (a) and (d) show the MSRE of the SM predictions of $\dot{\varepsilon}_{v m}$ for each validation case which falls in the subdomain. A single case is highlighted in (b) and (c), and another case is shown in (e) and (f). (b) and (e) show the new SM prediction of $\dot{\varepsilon}_{v m}(t)$ in blue with the VPSC result in red. (c) and (f) show the original $S M$ prediction in blue, with the same VPSC results in red. The high stress case had the input values, $\sigma_{v m}=257.8 \mathrm{MPa}, T=845.6 \mathrm{~K}, \rho_{\text {cell }}=6.473 \times 10^{12} \mathrm{~m}^{-2}$ and $\rho_{\text {wall }}=8.996 \times 10^{11} \mathrm{~m}^{-2}$, and the low stress case had the input values, $\sigma_{v m}=147.6 \mathrm{MPa}, T=972.3 \mathrm{~K}, \rho_{\text {cell }}=5.847 \times$ $10^{12} \mathrm{~m}^{-2}$ and $\rho_{\text {wall }}=5.476 \times 10^{11} \mathrm{~m}^{-2}$ 
Table 3 The optimized input mappings for the $\dot{\varepsilon}_{v m}$ predictions of the subdomain SMs. The output mappings are preserved from the original $S M$

Optimized Mappings for High $\sigma_{v m}$

\begin{tabular}{c|c|c|c|c|c}
\cline { 2 - 6 } Input: & $\varepsilon_{v m}^{*}$ & $\rho_{\text {cell }}^{*}\left(m^{-2}\right)$ & $\rho_{W}^{*}\left(m^{-2}\right)$ & $\sigma_{v m}^{*}(\mathrm{MPa})$ & $T^{*}(\mathrm{~K})$ \\
\hline$\dot{\varepsilon}_{v m}^{* *}()$ & $\frac{1}{\varepsilon_{v m}+c}$ & $e^{\frac{\rho_{\text {cell }}}{c}}$ & $e^{\frac{\rho_{w}}{c}}$ & $e^{\frac{\sigma_{v m}}{c}}$ & $e^{\frac{T}{c}}$ \\
\hline$c=$ & $1.91 \times 10^{-3}$ & $2.93 \times 10^{12}$ & $1.61 \times 10^{11}$ & 140.3 & 263.8 \\
\hline
\end{tabular}

Optimized Mappings for Low $\sigma_{v m}$

\begin{tabular}{c|c|c|c|c|c}
\hline Input: & $\varepsilon_{v m}^{*}$ & $\rho_{\text {cell }}^{*}\left(m^{-2}\right)$ & $\rho_{W}^{*}\left(m^{-2}\right)$ & $\sigma_{v m}^{*}(\mathrm{MPa})$ & $T^{*}(\mathrm{~K})$ \\
\hline$\dot{\varepsilon}_{v m}^{* *}()$ & $\ln \varepsilon_{v m}+c$ & $e^{\frac{\rho_{\text {cell }}}{c}}$ & $e^{\frac{\rho_{w}}{c}}$ & $\ln \sigma_{v m}+c$ & $e^{\frac{T}{c}}$ \\
\hline$c=$ & 10.80 & $1.78 \times 10^{12}$ & $4.51 \times 10^{11}$ & 279.8 & 42.76 \\
\hline
\end{tabular}

The marked improvement in the subdomain SM predictions of $\dot{\varepsilon}_{v m}$ is promising. It may be most effective to segment the target domain into a large number of subdomains. The goal of connecting to FEA solvers will require a single model to be used. Therefore, it will be necessary to develop a method to combine the results of these subdomain models. The development of this method is left for future work.

\section{Conclusions}

In this work a Surrogate Model (SM) for $316 \mathrm{H}$ stainless steel was developed for high temperature creep applications. The SM utilizes a database of simulation results from a CP-based constitutive model embedded in the crystal plasticity code VPSC. The constitutive model uses an internal state variable based mechanistic description of the deformation of $316 \mathrm{H}$ at high temperatures. The SM employs orthogonal polynomial regression to incorporate large volumes of data without escalating costs at runtime. Validation of the SM was performed using creep test 
simulations across a wide range of target operating conditions, and using additional loading cases which demonstrate the ability of the SM to capture transient responses. Runtimes for SM and full CP simulations typical differ by a factor of at least 10 . As a consequence, the SM can be used in the future in finite element component-scale creep simulations, at greatly reduced runtimes and comparable precision as provided by polycrystal models.

\section{Acknowledgements}

This work was sponsored by the U.S. Department of Energy, Office of Nuclear Energy, Nuclear Energy Advanced Modeling and Simulations (NEAMS). Los Alamos National Laboratory, an affirmative action/equal opportunity employer, is operated by Triad National Security, LLC, for the National Nuclear Security Administration of the U.S. Department of Energy under Contract No. 89233218CNA000001.

\section{Appendix}

Dislocation shear rate: Given by Eq. 6 from Section 2.1 is:

$$
\dot{\gamma}^{s}=\rho_{\text {cell }}^{s} b^{s} v^{s} \operatorname{sign}\left(\tau^{s}\right)
$$

here $\operatorname{sign}\left(\tau^{S}\right)$ defines the direction of the shear rate to be the same as the direction of glide. $b^{S}$ is the magnitude of the Burgers vector. $v^{s}$ is the mean dislocation velocity, which is calculated from the dislocation mean free path, $\lambda^{s}$, and the total time a dislocation spends traveling between obstacles. The total time is the sum of the waiting time at obstacles $\left(t_{w}^{s}\right)$ and the travel time within the interspacing $\left(t_{t}^{S}\right)$ :

$$
v^{s}=\frac{\lambda^{s}}{t_{w}^{s}+t_{t}^{s}}
$$


The mean dislocation interspacing associated with dislocation-dislocation interactions is given as in Franciosi and Zaoui [75,76]:

$$
\frac{1}{\lambda^{s}}=\sqrt{\sum_{S} \bar{\alpha}^{S S \prime} \rho_{c e l l}^{S \prime}}
$$

where $\bar{\alpha}^{S S \prime}$ refers to the latent hardening matrix. Using the Kocks-type activation enthalpy law [77-79], the dislocation waiting time is written as:

$$
t_{w}^{S}=\left\{\begin{array}{cl}
\frac{1}{v^{s}} \exp \left(\frac{\Delta G_{0}}{k T}\left(1-\left(\frac{\left|\tau_{\mathrm{eff}}^{s}\right|}{\tau_{c}^{S}}\right)^{p}\right)^{q}\right) & \text { if }\left|\tau^{S}\right|<\tau_{c}^{S} \\
0 & \text { if }\left|\tau^{S}\right| \geq \tau_{c}^{S}
\end{array}\right.
$$

here $\Delta G_{0}$ is the thermal activation energy without any external stress. $k$ is the Boltzmann constant. $T$ is absolute temperature. $p(0<p \leq 1)$ and $q(0<q \leq 2)$ are the exponent parameters related to the shape of the obstacle resistance profile [78]. $v^{s}=\chi_{e} C_{s} / \lambda^{S}$ is the attack frequency. Here, $C_{s}$ is the shear wave velocity and $\chi_{e}$ is an entropy factor (of the order of 1 ). $\tau_{c}^{S}$ refers to the Critical Resolved Shear Stress (CRSS), which is expressed via use of a non-linear superposition law [7981]:

$$
\tau_{c}^{S}=\tau_{0}^{S}+\left(\tau_{c w}^{n}+\tau_{h}^{n}\right)^{1 / n}
$$

where $\tau_{c w}=\mu b^{s} \sqrt{\sum_{s} \bar{\alpha}^{S S \prime} \rho_{c w}^{S \prime}}$ denotes the cell wall-induced hardening and $\tau_{h}$ denotes the strengthening due to solute pinning and precipitates. $\tau_{\text {eff }}^{S}$ in Eq. 10 represents the effective driving stress acting on dislocations inside the cells and it is expressed as:

$$
\tau_{\mathrm{eff}}^{s}=\tau^{S}-\Delta \tau_{m}^{S}-\Delta \tau_{l}^{S}
$$


where $\tau^{S}$ denotes the local resolved shear stress. $\Delta \tau_{m}^{S}$ and $\Delta \tau_{l}^{S}$ are associated with the local reduction in driving force acting on dislocations due to the presence of solutes and due to line tension respectively. As per Wen et al. [12], $\Delta \tau_{m}^{S}$ and $\Delta \tau_{l}^{S}$ are written as:

$$
\Delta \tau_{m}^{S}\left(t_{a, l o c a l}^{S}\right)=\frac{\alpha \Delta E^{\text {core }}\left(t_{a, l o c a l}^{s}\right)}{\bar{w} b^{s}} \text { and } \Delta \tau_{l}^{S}=\mu b^{s} \sqrt{\sum_{s} \bar{\alpha}^{S S \prime} \rho_{c e l l}^{S \prime}}
$$

$t_{a, l o c a l}^{s}$ is the local aging time (pinning period). $\alpha$ is associated with the energy variation along the core. $\bar{w}$ denotes the core width and $\Delta E^{\text {core }}$ is the binding energy of the solute to the dislocation.

Dislocation climb rate: Given by Eq. 7 of Section 2.1 is:

$$
\dot{\beta}^{s}=\rho_{\text {cell,edge }}^{s} b^{s} v_{\text {climb }}^{s}
$$

here $\rho_{\text {cell,edge }}^{S}$ denotes the edge dislocation density. In the present work $\rho_{\text {cell,edge }}^{S}=0.1 \rho_{\text {cell }}^{S}$ is assumed. $v_{\text {climb }}^{s}$ in Eq. A8 represents the climb velocity, which depends on the net flux of point defects $I^{s}$. The climb velocity $v_{\text {climb }}^{S}$ depends on the imbalance between vacancies and interstitials being trapped by the dislocation, which can be written using a classic expression of rate theory, as:

$$
\bar{v}_{c}^{s}=\frac{\Omega}{b}\left(z_{v}^{s} D_{v} c_{v}^{t h}\left[\exp \left(\frac{\Omega \bar{\tau}_{c l i m b}^{s}}{k T}\right)-1\right]\right)
$$

where $D_{v}=D_{v}^{0} \exp \left(-E_{m}^{v} / k T\right)$ denotes the vacancy diffusivity and $z_{v}^{s}$ is the rate-theory parameter representing the dislocation capture efficiency for vacancies. $\Omega \approx b^{3}$ represents the atomic volume and $c_{v}^{\text {th }}$ is the thermal equilibrium vacancy concentration. 
Dislocation density evolution: A recently developed dislocation density law for Fe-Cr-Mo alloy is employed here [61]. As mentioned before, the dislocation content can be divided into two populations: dislocations in the cell (subgrain) and in the cell walls (subgrain boundary). The evolution of the dislocation density in the cell is expressed as:

$$
\dot{\rho}_{\text {cell }}^{s}=\dot{\rho}_{\text {cell,g }}^{s,+}-\dot{\rho}_{\text {cell,a }}^{s,-}-\dot{\rho}_{\text {cell,trap }}^{s,-}
$$

Where $\dot{\rho}_{\text {cell,g }}^{s,+}, \dot{\rho}_{\text {cell,a, }}^{s,-}$, and $\dot{\rho}_{\text {cell,trap }}^{s,-}$ denote the dislocation generation, dynamic recovery and trapping at the subgrain boundaries. The dislocation generation rate is associated with the area swept by the moving dislocations. The term $\dot{\rho}_{\text {cell,g }}^{s,+}$ can be expressed as [82]:

$$
\dot{\rho}_{\text {cell,g }}^{s,+}=\frac{k_{1}}{b \lambda^{s}}\left|\overline{\dot{\gamma}}^{s}\right|
$$

Where $\frac{\lambda^{s}}{k_{1}}$ is the effective mean free path. The dynamic recovery involves several mechanisms, such as cross-slip and climb, that allow the dislocation to move to another slip plane and annihilate with dislocations with opposite Burger vector. Estrin [83] proposed a general expression of the dynamic recovery rate:

$$
\dot{\rho}_{\text {cell,a }}^{s,-}=k_{2}\left(\frac{\dot{\varepsilon}_{0}}{\dot{\varepsilon}}\right)^{\frac{1}{n_{0}}} \rho_{\text {cell }}^{s}\left|\overline{\dot{\gamma}}^{s}\right|
$$

where $\dot{\varepsilon}_{0}$ is a reference strain rate. Estrin suggested that the parameter $n_{0}$ should be associated with the dominant mechanism and it can vary between 3 and 5 [83]. The dislocation trapping rate at the subgrain boundaries is related to the subgrain size $\lambda_{\mathrm{sg}}$ :

$$
\dot{\rho}_{\text {cell,trap }}^{s,-}=\frac{k_{3}}{\lambda_{\text {sg }}}\left|\overline{\dot{\gamma}}^{s}\right|
$$


The trapped dislocations will essentially become part of the wall structure. Meanwhile, the dislocations in the cell wall will also annihilate. Thus, the rate of $\rho_{c w}^{S}$ can be written as:

$$
\dot{\rho}_{\mathrm{cW}}^{S}=\dot{\rho}_{\text {cell,trap }}^{s,-}-\dot{\rho}_{\mathrm{cw}, \mathrm{a}}^{s,-}
$$

Dislocation annihilation in the subgrain boundaries is complex and for the sake of simplicity, the annihilation rate is written as:

$$
\dot{\rho}_{\mathrm{cw}, \mathrm{a}}^{s,-}=k_{4} \rho_{c w}^{s}\left|\overline{\dot{\gamma}}^{s}\right|
$$

The parameters $k_{1}, k_{2}, k_{3}$ and $k_{4}$ are material constants calibrated using the experimental data.

Relative activity of deformation mechanisms: Relative contribution of glide, climb and Coble creep modes to the predicted creep responses is shown in Fig. 10 as a function of imposed stress for three different temperatures. At the early stages of creep, dislocation glide dominates the deformation for all the stress and temperature cases. Within a few hours, contribution of glide decreases and, depending on the stress and temperature, climb and Coble creep modes starts to activate. The relative activity of climb increases with the temperature and decreases with increasing imposed stress. Similarly, the contribution of Coble creep also decreases with imposed stress.

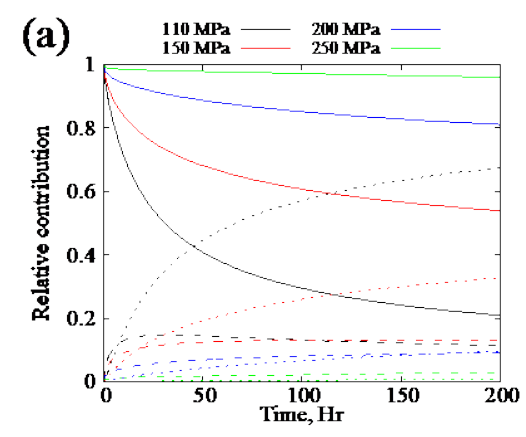

Solid: Glide

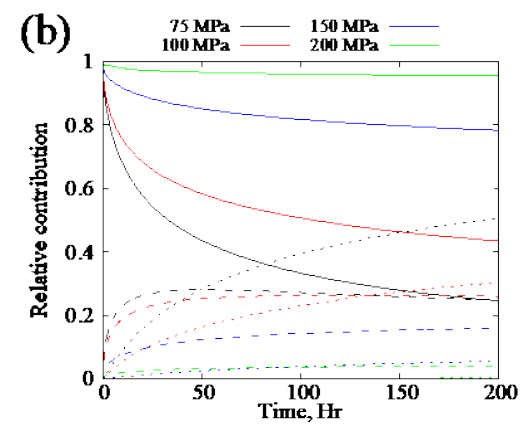

Dashed: Climb

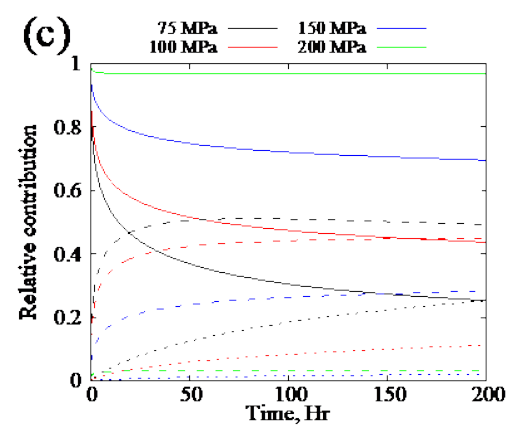

Dotted: Coble creep 
Fig. 10. Relative contribution of individual deformation mechanisms (glide, climb and Coble creep) as a function of imposed stress for (a) $650{ }^{\circ} \mathrm{C}$, (b) $700{ }^{\circ} \mathrm{C}$ and (c) $750{ }^{\circ} \mathrm{C}$. Contribution of climb increases with temperature. The relative activity of both the climb and Coble creep modes are inversely proportional to imposed stress.

Table 4. The calibrated constitutive model parameter values for $316 \mathrm{H}$ steel. (* parameter estimated or back fitted from experimental data). Refer Wen et al. [61] for fitted parameter ranges and details for the other parameters.

\begin{tabular}{|c|c|c|c|}
\hline Parameters & Values & Parameters & Values \\
\hline $\begin{array}{c}b \text { (magnitude of Burgers } \\
\text { vector) }\end{array}$ & $2.546 \cdot 10^{-10} \mathrm{~m}$ & $\lambda_{s g}($ sub-grain size $)$ & $0.5 \cdot 10^{-7}$ \\
\hline$\mu$ (shear modulus) & $\begin{array}{c}87828 \mathrm{MPa} \\
-T \cdot 36 \mathrm{MPa} / \mathrm{K}\end{array}$ & $\begin{array}{c}n_{0} \text { (annihilation strain rate } \\
\text { sensitivity)* }\end{array}$ & 3.5 \\
\hline$\tau_{0}$ (friction stress) & $0 \mathrm{MPa}$ & $\dot{\varepsilon}_{0}($ reference strain rate $)$ & $1 \mathrm{~s}^{-1}$ \\
\hline $\begin{array}{c}\tau_{h} \text { (hardening contribution } \\
\text { from solute pinning and } \\
\text { precipitation)* }\end{array}$ & $\begin{array}{c}957 \mathrm{MPa} \\
-T \cdot 0.6 \mathrm{MPa} / \mathrm{K}\end{array}$ & $n$ (superposition power) & 2.0 \\
\hline$\eta$ (scaling parameter)* & $3.8 \cdot 10^{-6} \mathrm{MPa} / \mathrm{m}$ & $\varphi$ (power parameter) & 1 \\
\hline $\begin{array}{l}\Delta G_{0} \text { (zero-stress activation } \\
\text { energy for dislocations)* }\end{array}$ & $3 \mathrm{eV}$ & $\bar{w}$ (width of dislocation core) & $1.86 \mathrm{~nm}$ \\
\hline$p$ (exponent parameter) $^{*}$ & 0.7 & $\begin{array}{l}\chi \text { (pre-factor linking binding } \\
\text { energy and junction strength) }\end{array}$ & 12 \\
\hline$q$ (exponent parameter) $^{*}$ & 1.4 & $\alpha\left(\right.$ scaling coefficient for $\left.\Delta \tau_{m}^{s}\right)$ & 0.38 \\
\hline$\chi_{e}$ (entropy factor) & 0.5 & $\begin{array}{l}\alpha_{0}^{S \prime}(\text { saturation dislocation- } \\
\text { dislocation interaction) }\end{array}$ & $\begin{array}{l}0.7\left(s=s^{\prime}\right) \\
0.05\left(s \neq s^{\prime}\right)\end{array}$ \\
\hline $\begin{array}{l}\rho_{\text {cell, }, 0}^{S} \text { (initial dislocation } \\
\text { density in the cell) }\end{array}$ & $1 \cdot 10^{13} \mathrm{~m}^{-2}$ & $D_{v}^{0}($ vacancy diffusion constant $)$ & $1.5 \cdot 10^{-4} \mathrm{~m}^{2} \mathrm{~s}^{-1}$ \\
\hline $\begin{array}{c}\rho_{W, 0}^{S} \text { (initial dislocation } \\
\text { density in the cell wall) }\end{array}$ & $5 \cdot 10^{11} \mathrm{~m}^{-2}$ & $E_{m}^{v}($ vacancy migration energy) & $1.1 \mathrm{eV}$ \\
\hline$k_{1}$ (material constant)* $^{*}$ & 0.09 & $E_{f}^{v}$ (vacancy formation energy) & $2.0 \mathrm{eV}$ \\
\hline$k_{2}{\text { (material constant })^{*}}$ & 45 & $S_{f}^{v}$ (vacancy formation entropy) & $3.6 \cdot 10^{-4} \mathrm{eV} / \mathrm{K}$ \\
\hline$k_{3}$ (material constant)* & $1.0 \cdot 10^{8}$ & $A_{\text {coble }}(\text { coble creep parameter })^{*}$ & $5 \cdot 10^{-8}$ \\
\hline$k_{4}$ (material constant)* & 800 & $Q_{g b}$ (coble creep parameter)* & $0.07 \mathrm{eV}$ \\
\hline
\end{tabular}


LHSMDU Example: The two samples shown below (Fig. 11) portray the effective difference in the sampling method employed here and a simple Monte-Carlo random sampling.
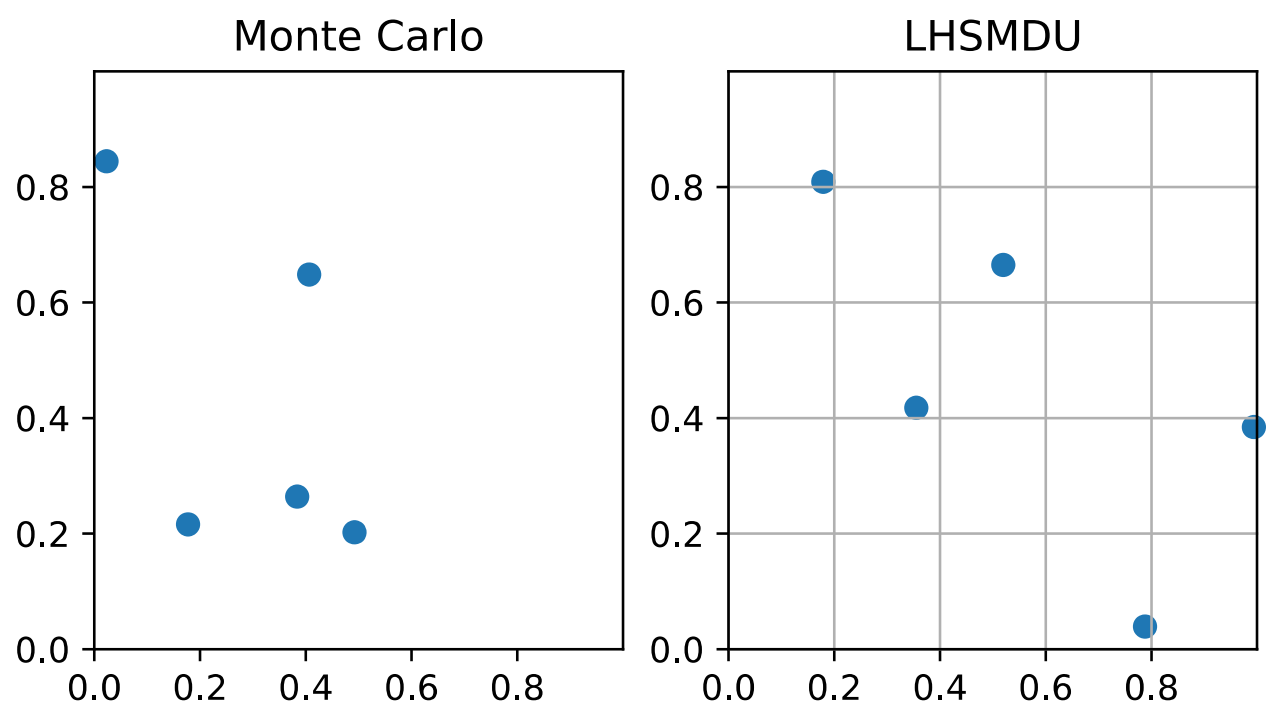

Fig. 11. A comparison of a Monte Carlo (random) sampling with a LHSMDU sampling in 2 dimensions. The average minimum distance between points for the two method examples are 0.25 and 0.35 , respectively

Uncertainty from anisotropy: The error induced with the assumption of an isotropic polycrystal is quantified using a batch of $20 \mathrm{CP}$-VPSC simulations, run with the same von Mises stress, temperature, microstructure, and initial dislocation densities $\left(\sigma_{v m}=300 \mathrm{MPa}, T=\right.$ $973.0 \mathrm{~K}, \rho_{\text {cell }}=7.0 \times 10^{12} \mathrm{~m}^{-2}$ and $\rho_{\text {wall }}=7.0 \times 10^{11} \mathrm{~m}^{-2}$ ), only varying the relative contribution of individual stress tensor components. The results of this set of VPSC simulations are shown in Fig. 12. The effective strain of each simulation is shown as a function of time. It can be seen that the simulations present variability in the strain rate resulting from the same level of stress. The variability would decrease if an increasing number of orientations were considered for representing the aggregate (50 orientations were used in the calculation). The low relative scatter in the simulations is used to support the $\mathrm{J} 2$ assumption. 


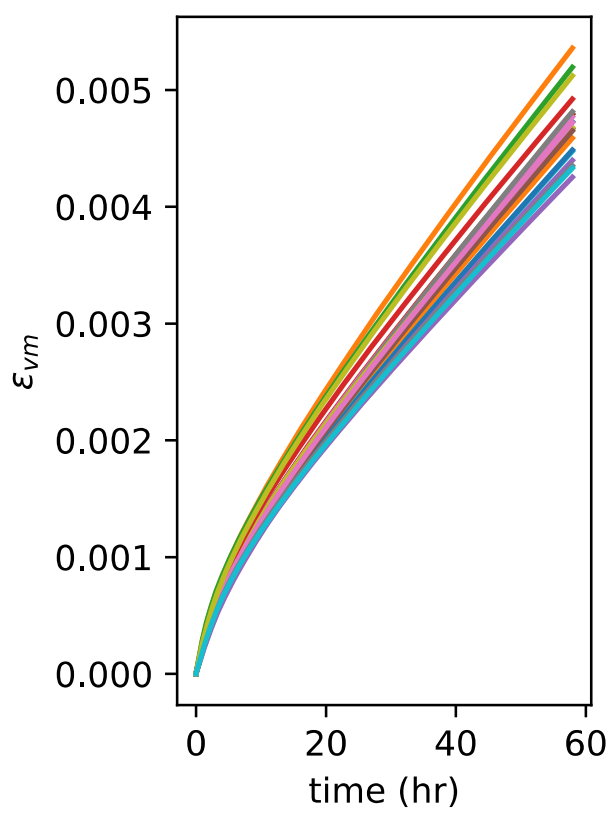

Fig. 12. The CP simulations made to quantify the uncertainty associated with the use of a PrandtlReuss flow rule in the SM formulation in predicted plastic strain. Each solid line reflects a different stress direction with respect to a fixed microstructure orientation. Initial parameters are, for all runs: $\sigma_{v m}=300 \mathrm{MPa}, T=973.0 \mathrm{~K}, \rho_{\text {cell }}=7.0 \times 10^{12} \mathrm{~m}^{-2}$ and $\rho_{\text {wall }}=7.0 \times 10^{11} \mathrm{~m}^{-2}$

\section{References}

[1] Lee M G, Kim S J, Wagoner R H, Chung K and Kim H Y 2009 Constitutive modeling for anisotropic/asymmetric hardening behavior of magnesium alloy sheets: Application to sheet springback Int. J. Plast. 25 70-104

[2] Chaboche J L 2008 A review of some plasticity and viscoplasticity constitutive theories Int. J. Plast. 24 1642-93

[3] Chen B, Smith D J, Flewitt P E J and Spindler M W 2011 Constitutive equations that describe creep stress relaxation for $316 \mathrm{H}$ stainless steel at $550^{\circ} \mathrm{C}$ Mater. High Temp. 28 155-64

[4] Hyde T H, Becker A A, Sun W and Williams J A 2006 Finite-element creep damage analyses of P91 pipes Int. J. Press. Vessels Pip. 83 853-63

[5] Goyal S, Laha K, Das C R, Panneer Selvi S and Mathew M D 2013 Finite element analysis of uniaxial and multiaxial state of stress on creep rupture behaviour of $2.25 \mathrm{Cr}-1 \mathrm{Mo}$ steel Mater. Sci. Eng. A 563 68-77 
[6] Hall F R and Hayhurst D R 1991 Continuum Damage Mechanics Modelling of High Temperature Deformation and Failure in a Pipe Weldment Proc. Math. Phys. Sci. 433 383403

[7] Frost H J and Ashby M F 1977 Deformation-Mechanism Maps for Pure Iron, Two Austenitic Stainless Steels, and a Low-Alloy Ferritic Steel Fundamental Aspects of Structural Alloy Design Battelle Institute Materials Science Colloquia ed R I Jaffee and B A Wilcox (Boston, MA: Springer US) pp 27-65

[8] Chen B, Flewitt P E J, Cocks A C F and Smith D J 2015 A review of the changes of internal state related to high temperature creep of polycrystalline metals and alloys Int. Mater. Rev. $601-29$

[9] Wang Y-J, Ishii A and Ogata S 2011 Transition of creep mechanism in nanocrystalline metals Phys. Rev. B $\mathbf{8 4} 224102$

[10] Yang X-S, Wang Y-J, Zhai H-R, Wang G-Y, Su Y-J, Dai L H, Ogata S and Zhang T-Y 2016 Time-, stress-, and temperature-dependent deformation in nanostructured copper: Creep tests and simulations J. Mech. Phys. Solids 94 191-206

[11] Kloc L and Sklenička V 1997 Transition from power-law to viscous creep behaviour of p91 type heat-resistant steel Mater. Sci. Eng. A 234-236 962-5

[12] Wen W, Kohnert A, Arul Kumar M, Capolungo L and Tomé C N 2020 Mechanism-based modeling of thermal and irradiation creep behavior: An application to ferritic/martensitic HT9 steel Int. J. Plast. 126102633

[13] Kloc L, Skienička V and Ventruba J 2001 Comparison of low stress creep properties of ferritic and austenitic creep resistant steels Mater. Sci. Eng. A 319-321 774-8

[14] Pahutová M 1980 Research Report UFM CSAV (Brno)

[15] Rabotnov Yu N 1965 Experimental data on creep of engineering alloys and phenomenological theories of creep. A review J. Appl. Mech. Tech. Phys. 6 137-54

[16] Wilshire B and Scharning P J 2008 Extrapolation of creep life data for $1 \mathrm{Cr}-0.5 \mathrm{Mo}$ steel Int. J. Press. Vessels Pip. 85 739-43

[17] Brown S B, Kim K H and Anand L 1989 An internal variable constitutive model for hot working of metals Int. J. Plast. 5 95-130

[18] Chaboche J L 1989 Constitutive equations for cyclic plasticity and cyclic viscoplasticity Int. J. Plast. 5 247-302

[19] Watanabe O and Atluri S N 1986 Constitutive modeling of cyclic plasticity and creep, using an internal time concept Int. J. Plast. 2 107-34 
[20] Murakami S and Ohno N 1982 A constitutive equation of creep based on the concept of a creep-hardening surface Int. J. Solids Struct. 18 597-609

[21] Moosbrugger J C and McDowell D L 1989 On a Class of Kinematic Hardening Rules for Nonproportional Cyclic Plasticity J. Eng. Mater. Technol. 111 87-98

[22] Bammann D J 1984 An internal variable model of viscoplasticity Int. J. Eng. Sci. 22 104153

[23] Bammann D J 1990 Modeling Temperature and Strain Rate Dependent Large Deformations of Metals Appl. Mech. Rev. 43 S312-9

[24] Johnson G R and Cook W H 1983 A constitutive model and data for materials subjected to large strains, high strain rates, and high temperatures Proc 7th Inf Sympo Ballist. 541-547

[25] Mecking H and Kocks U F 1981 Kinetics of flow and strain-hardening Acta Metall. 29 186575

[26] Norton F H 1929 The creep of steel at high temperatures (New York [etc.]: McGraw-Hill book company, inc.)

[27] Garofalo F 1963 An Empirical Relation Defining Stress Dependence of Minimum Creep Rate Trans. Metall. Soc. Aime 227 351-

[28] Mukherjee A, Bird J and Dorn J 1969 Experimental Correlations for High-Temperature Creep Asm Trans. Q. 62 155-+

[29] Bari S and Hassan T 2002 An advancement in cyclic plasticity modeling for multiaxial ratcheting simulation Int. J. Plast. 18 873-94

[30] Lu Z K and Weng G J 1996 A simple unified theory for the cyclic deformation of metals at high temperature Acta Mech. 118 135-49

[31] Nouailhas D 1989 Unified modelling of cyclic viscoplasticity: Application to austenitic stainless steels Int. J. Plast. 5 501-20

[32] Tanaka E 1994 A Nonproportionality Parameter and a Cyclic Viscoplastic Constitutive Model Taking into Account Amplitude Dependences and Memory Effects of Isotropic Hardening Eur. J. Mech. -Solids 13 155-73

[33] Keralavarma S M, Cagin T, Arsenlis A and Benzerga A A 2012 Power-Law Creep from Discrete Dislocation Dynamics Phys. Rev. Lett. 109265504

[34] Patra A and McDowell D L 2012 Crystal plasticity-based constitutive modelling of irradiated bcc structures Philos. Mag. 92 861-87

[35] Beyerlein I J and Tomé C N 2008 A dislocation-based constitutive law for pure $\mathrm{Zr}$ including temperature effects Int. J. Plast. 24 867-95 
[36] Krishna S, Zamiri A and De S 2010 Dislocation and defect density-based micromechanical modeling of the mechanical behavior of fcc metals under neutron irradiation Philos. Mag. 90 4013-25

[37] Needleman A, Asaro R J, Lemonds J and Peirce D 1985 Finite element analysis of crystalline solids Comput. Methods Appl. Mech. Eng. 52 689-708

[38] Asaro R J 1983 Crystal Plasticity J. Appl. Mech. 50 921-34

[39] Roters F, Eisenlohr P, Hantcherli L, Tjahjanto D D, Bieler T R and Raabe D 2010 Overview of constitutive laws, kinematics, homogenization and multiscale methods in crystal plasticity finite-element modeling: Theory, experiments, applications Acta Mater. 58 1152-211

[40] Roters F, Raabe D and Gottstein G 2000 Work hardening in heterogeneous alloys-a microstructural approach based on three internal state variables Acta Mater. 48 4181-9

[41] Barton N R, Arsenlis A and Marian J 2013 A polycrystal plasticity model of strain localization in irradiated iron J. Mech. Phys. Solids $61341-51$

[42] Castelluccio G M and McDowell D L 2017 Mesoscale cyclic crystal plasticity with dislocation substructures Int. J. Plast. 98 1-26

[43] Arsenlis A, Wirth B D and Rhee M 2004 Dislocation density-based constitutive model for the mechanical behaviour of irradiated Cu Philos. Mag. 84 3617-35

[44] Nes E 1997 Modelling of work hardening and stress saturation in FCC metals Prog. Mater. Sci. 41 129-93

[45] Coble R L 1963 A Model for Boundary Diffusion Controlled Creep in Polycrystalline Materials J. Appl. Phys. 34 1679-82

[46] Lebensohn R A, Hartley C S, Tomé C N and Castelnau O 2010 Modeling the mechanical response of polycrystals deforming by climb and glide Philos. Mag. 90 567-83

[47] Bishop J E, Emery J M, Field R V, Weinberger C R and Littlewood D J 2015 Direct numerical simulations in solid mechanics for understanding the macroscale effects of microscale material variability Comput. Methods Appl. Mech. Eng. 287 262-89

[48] Miehe C, Schröder J and Schotte J 1999 Computational homogenization analysis in finite plasticity Simulation of texture development in polycrystalline materials Comput. Methods Appl. Mech. Eng. 171 387-418

[49] Knezevic M and Savage D J 2014 A high-performance computational framework for fast crystal plasticity simulations Comput. Mater. Sci. 83 101-6

[50] Zecevic M, McCabe R J and Knezevic M 2015 Spectral database solutions to elastoviscoplasticity within finite elements: Application to a cobalt-based FCC superalloy Int. J. Plast. 70 151-65 
[51] Smit R J M, Brekelmans W A M and Meijer H E H 1998 Prediction of the mechanical behavior of nonlinear heterogeneous systems by multi-level finite element modeling Comput. Methods Appl. Mech. Eng. 155 181-92

[52] Patra A and Tomé C N 2017 Finite element simulation of gap opening between cladding tube and spacer grid in a fuel rod assembly using crystallographic models of irradiation growth and creep Nucl. Eng. Des. 315 155-69

[53] Forrester A, Sobester A and Keane A 2008 Engineering Design Via Surrogate Modelling: A Practical Guide (Chichester, West Sussex, England: Wiley-Blackwell)

[54] Fast T and Kalidindi S R 2011 Formulation and calibration of higher-order elastic localization relationships using the MKS approach Acta Mater. 59 4595-605

[55] Becker R and Lloyd J T 2016 A reduced-order crystal model for HCP metals: Application to $\mathrm{Mg}$ Mech. Mater. 98 98-110

[56] Kalidindi S R, Duvvuru H K and Knezevic M 2006 Spectral calibration of crystal plasticity models Acta Mater. 54 1795-804

[57] Knezevic M, Al-Harbi H F and Kalidindi S R 2009 Crystal plasticity simulations using discrete Fourier transforms Acta Mater. 57 1777-84

[58] Zecevic M, McCabe R J and Knezevic M 2015 A new implementation of the spectral crystal plasticity framework in implicit finite elements Mech. Mater. 84 114-26

[59] Narula S C 1979 Orthogonal Polynomial Regression Int. Stat. Rev. Rev. Int. Stat. 47 31-6

[60] Wang H, Capolungo L, Clausen B and Tomé C N 2017 A crystal plasticity model based on transition state theory Int. J. Plast. 93 251-68

[61] Wen W, Capolungo L, Patra A and Tomé C N 2017 A Physics-Based Crystallographic Modeling Framework for Describing the Thermal Creep Behavior of Fe-Cr Alloys Metall. Mater. Trans. A 48 2603-17

[62] Wang H, Clausen B, Capolungo L, Beyerlein I J, Wang J and Tomé C N 2016 Stress and strain relaxation in magnesium AZ31 rolled plate: In-situ neutron measurement and elastic viscoplastic polycrystal modeling Int. J. Plast. 79 275-92

[63] Wen W, Capolungo L and Tomé C N 2018 Mechanism-based modeling of solute strengthening: Application to thermal creep in $\mathrm{Zr}$ alloy Int. J. Plast. 106 88-106

[64] Lebensohn R A and Tomé C N 1993 A self-consistent anisotropic approach for the simulation of plastic deformation and texture development of polycrystals: Application to zirconium alloys Acta Metall. Mater. 41 2611-24 
[65] Lebensohn R A, Tomé C N and CastaÑeda P P 2007 Self-consistent modelling of the mechanical behaviour of viscoplastic polycrystals incorporating intragranular field fluctuations Philos. Mag. 87 4287-322

[66] Hill R 1967 The essential structure of constitutive laws for metal composites and polycrystals J. Mech. Phys. Solids 15 79-95

[67] Asaro R J and Rice J R 1977 Strain localization in ductile single crystals J. Mech. Phys. Solids 25 309-38

[68] Joseph V R 2016 Space-filling designs for computer experiments: A review Qual. Eng. 28 $28-35$

[69] Pronzato L and Müller W G 2012 Design of computer experiments: space filling and beyond Stat. Comput. 22 681-701

[70] Tang B 1993 Orthogonal Array-Based Latin Hypercubes J. Am. Stat. Assoc. 88 1392-7

[71] Deutsch J L and Deutsch C V 2012 Latin hypercube sampling with multidimensional uniformity J. Stat. Plan. Inference 142 763-72

[72] Moza S 2019 sahilm89/lhsmdu: First release for this code (Zenodo)

[73] Khuri A I and Mukhopadhyay S 2010 Response surface methodology Wiley Interdiscip. Rev. Comput. Stat. 2 128-49

[74] Weisstein E W 2002 Legendre Polynomial MathWorld-- Wolfram Web Resour.

[75] Franciosi P and Zaoui A 1982 Multislip in f.c.c. crystals a theoretical approach compared with experimental data Acta Metall. 30 1627-37

[76] Franciosi P and Zaoui A 1982 Multislip tests on copper crystals: A junctions hardening effect Acta Metall. 30 2141-51

[77] Kocks U F, Argon A S and Ashby M F 1975 Thermodynamics and Kinetics of Slip (Pergamon Press)

[78] Austin R A and McDowell D L 2011 A dislocation-based constitutive model for viscoplastic deformation of fcc metals at very high strain rates Int. J. Plast. 27 1-24

[79] Lloyd J T, Clayton J D, Austin R A and McDowell D L 2014 Plane wave simulation of elastic-viscoplastic single crystals J. Mech. Phys. Solids 69 14-32

[80] Dong Y, Nogaret T and Curtin W A 2010 Scaling of Dislocation Strengthening by Multiple Obstacle Types Metall. Mater. Trans. A 41 1954-60

[81] Lagerpusch U, Mohles V, Baither D, Anczykowski B and Nembach E 2000 Double strengthening of copper by dissolved gold-atoms and by incoherent SiO2-particles: how do the two strengthening contributions superimpose? Acta Mater. 48 3647-56 
[82] Kitayama K, Tomé C N, Rauch E F, Gracio J J and Barlat F 2013 A crystallographic dislocation model for describing hardening of polycrystals during strain path changes. Application to low carbon steels Int. J. Plast. 46 54-69

[83] Estrin Y 1998 Dislocation theory based constitutive modelling: foundations and applications J. Mater. Process. Technol. 80-81 33-9 